\title{
Evaluation der Implementation konzeptuell unterschiedlicher Maßnahmen zur Leseförderung in der Sekundarstufe I
}

\author{
Elmar Souvignier • Nina Zeuch (D) · Jörg Jost • Fabiana Karstens • \\ Sarah-Ines Meudt • Anke Schmitz
}

Eingegangen: 16. Oktober 2020 / Überarbeitet: 30. April 2021 / Angenommen: 21. Mai 2021 / Online publiziert: 23. Juli 2021

(C) Der/die Autor(en) 2021

Zusammenfassung Im vorliegenden Beitrag werden basierend auf einem Evaluationsprojekt im Rahmen des von Bund und Ländern geförderten Programms „Bildung durch Sprache und Schrift“" (BiSS) Analysen zu Bedingungen einer wirksamen Implementation von evidenzbasierten Lesefördermaßnahmen in den schulischen Alltag am Beispiel zweier BiSS-Verbünde und einer Kontrollgruppe vorgestellt. Sowohl hinsichtlich der Implementationsstrategie (top-down vs. bottom-up) als auch im Hinblick auf das Fördermaterial (einfach vs. komplex) unterschieden sich die beiden Fördermaßnahmen deutlich voneinander. Zur Evaluation der Implementation kamen Lehrkräftebefragungen und Kompetenztestungen von Schüler*innen zum Einsatz.

Prof. Dr. Elmar Souvignier · Dr. Nina Zeuch $(\varangle) \cdot$ Dr. Sarah-Ines Meudt

Institut für Psychologie in Bildung und Erziehung, Westfälische Wilhelms-Universität Münster,

Fliednerstr. 21, 48149 Münster, Deutschland

E-Mail: nina.zeuch@uni-muenster.de

Prof. Dr. Elmar Souvignier

E-Mail: elmar.souvignier@uni-muenster.de

Dr. Sarah-Ines Meudt

E-Mail: sarah-ines.meudt@uni-muenster.de

Prof. Dr. Jörg Jost · Fabiana Karstens

Institut für deutsche Sprache und Literatur II, Universität zu Köln,

Albertus-Magnus-Platz, 50923 Köln, Deutschland

Prof. Dr. Jörg Jost

E-Mail: joerg.jost@uni-koeln.de

Fabiana Karstens

E-Mail: fabiana.karstens@uni-koeln.de

Prof. Dr. Anke Schmitz

Institut Deutsche Sprache und Literatur und ihre Didaktik, Leuphana Universität Lüneburg,

Universitätsallee 15, 21335 Lüneburg, Deutschland

E-Mail: anke.schmitz@leuphana.de 
Zwei Stichproben von 100 bzw. 98 Lehrkräften wurden zu Begleitfaktoren der Leseförderung (Einstellungen, Selbstwirksamkeit, Wissen, Akzeptanz) und gewünschten Effekten (Wiedergabetreue, Nutzung) befragt. Im Rahmen einer Längsschnittuntersuchung an einer Stichprobe von $N=1054$ Schüler*innen wurden Entwicklungen wesentlicher Facetten der Lesekompetenz (Leseverständnis, Leseflüssigkeit, Lesestrategien, Lesemotivation) betrachtet. Die Ergebnisse zeigen zunächst, dass eine breite Nutzung und positive Effekte in den beteiligten Verbünden nicht in dem gewünschten Maße resultieren. Dabei liegen zwischen den beiden Verbünden - trotz der unterschiedlichen Fördermaßnahmen - nur marginale Unterschiede vor. Analysen der Interkorrelationsmuster zu den Implementationsmerkmalen weisen darauf hin, dass eine erfolgreiche Implementation letztlich von einer hohen Passung zwischen Merkmalen auf Seiten der Maßnahme, der Lehrkraft und der schulischen Rahmenbedingungen abhängt. Implikationen für Forschung und Praxis werden abgeleitet.

Schlüsselwörter Evaluation · Implementation · Leseförderung · Lesekompetenz

\section{Evaluation of the implementation of conceptually different programs to foster reading in middle school}

Abstract Based on an evaluation project as part of the program "Bildung durch Sprache und Schrift" (BiSS) funded by the German Federal Ministry of Education and Research, this study examines preconditions of effective implementation of reading skill promotion into school practice within two BiSS-groups and one control group. The two programs largely differed with respect to their implementation strategy (top-down vs. bottom-up) and complexity of the teaching material. We used teacher surveys and competence tests for students to evaluate the two programs. Two samples of 100 and 98 teachers were surveyed regarding preconditions (beliefs, self-efficacy, knowledge, acceptance) and regarding desired effects (fidelity, usage of materials). Development of students' reading skills (reading fluency, reading comprehension, reading strategies, reading motivation) was investigated in a longitudinal study with $N=1054$ students. Results reveal few usage and positive effects within the BiSS-groups than intended. Rather, contrasts between the results for the two programs are marginal despite their conceptual differences. Analyses of intercorrelations between aspects of implementation demonstrate that successful implementation depends on the fit of characteristics of the program, the individual teacher, and specific circumstances of the school. Implications for research and practice are discussed.

Keywords Evaluation - Implementation · Promotion of reading skills · Reading Competence 


\section{Einleitung}

Eine Vielzahl von Interventionsstudien gibt Hinweise darauf, wie evidenzbasierte Leseförderung prinzipiell gestaltet werden kann: Als besonders bewährte Methoden und Prinzipien gelten Lautleseverfahren zur Förderung der Leseflüssigkeit und die explizite Vermittlung von Lesestrategiewissen zur Förderung des Leseverständnisses, wobei sich kooperative Lernarrangements und das Lernen am Modell als wirksame Organisations- und Instruktionsformate erwiesen haben (Hattie 2009; Kamil et al. 2008; NICHD 2000; Slavin et al. 2008). Als theoretisches Rahmenkonzept wird dabei häufig auf das Konzept selbstregulierten Lernens zurückgegriffen (Dignath und Büttner 2008; Zimmerman 2000). Resümierend stellt Schneider (2019) in einem Übersichtsbeitrag fest, dass in den letzten drei bis vier Jahrzehnten eine größere Zahl nachweislich wirksamer Förderprogramme entwickelt wurde, dass allerdings Probleme beim Transfer solcher wirksamen Programme in die schulische Praxis festzustellen sind (vgl. auch Goldenbaum 2013; Gräsel 2010; Hasselhorn et al. 2014; Philipp und Souvignier 2016).

Ziel dieses Beitrags ist es, Bedingungen einer gelingenden Implementation evidenzbasierter Maßnahmen zur Leseförderung in den schulischen Alltag zu untersuchen. Die vorliegende Studie wurde im Rahmen des von Bund und Ländern geförderten Programms „Bildung durch Sprache und Schrift“ (BiSS) durchgeführt. Zwei zentrale Kriterien für die gelungene Implementation einer Maßnahme in die schulische Praxis sind, dass die Maßnahme breite Nutzung erfährt und dass sie zu positiven Effekten führt. Konkret wurden in dem BiSS-Evaluationsprojekt EILe die Nutzung von Lesefördermaßnahmen und die Frage nach Bedingungen für die Nutzung anhand der Befragung von Lehrkräften untersucht und es wurden Effekte auf die Entwicklung von Schüler*innenleistungen über zwei Schuljahre hinweg geprüft.

Während der Begriff der Implementation letztlich auf das Ziel einer hohen Verbreitung und nachhaltigen Nutzung ausgerichtet ist, wird mit dem Begriff des Transfers vor allem der Prozess des Übergangs innovativer Konzepte beschrieben, beispielsweise von der wissenschaftlichen Entwicklung in die (schulische) Anwendungspraxis (Gräsel 2010; Prenzel 2010). In diesem Sinne werden in der Folge die beiden Begriffe der Implementation und des Transfers jeweils verwendet, wenn in höherem Maße ein erwünschter Zielzustand (Implementation) oder der Prozess des Übergangs (Transfer) angesprochen werden.

\section{Theorie und Forschungsstand}

\subsection{Leseförderung in der Sekundarstufe in zwei BiSS-Verbünden}

Das BiSS-Programm bietet einen sehr praxisnahen Rahmen zur Evaluation der Implementation evidenzbasierter Konzepte zur Leseförderung. Regionale Verbünde von Schulen, die ein einheitliches Förderkonzept einsetzten, konnten sich an dem BiSSProgramm beteiligen und - optional - einer begleitenden Evaluation zustimmen. Die Evaluation erfolgte entsprechend in bestehenden Verbünden, die mit selbst gewählten Materialien arbeiteten. In dem Evaluationsprojekt BiSS-EILe konnten zwei 
Tab. 1 Die beiden Verbünde und ihre Konzepte im Vergleich. (Aus Zeuch et al. 2018)

\begin{tabular}{|c|c|c|}
\hline & $\begin{array}{l}\text { Niemanden zurücklassen (NZL) } \\
\text { Lesen macht stark }\end{array}$ & $\begin{array}{l}\text { Oberfranken }(\mathrm{OF}) \\
\text { Lesestrategiefächer }\end{array}$ \\
\hline Ziele & $\begin{array}{l}\text { Lesemotivation und selbstreguliertes Lesen, } \\
\text { sprachliche Bildung im Fach }\end{array}$ & Selbstreguliertes Lesen im Fach \\
\hline Einsatz & Deutsch- und Fachunterricht, Sekundarstufe I & $\begin{array}{l}\text { Deutsch- und Fachunterricht, } \\
\text { Sekundarstufe I }\end{array}$ \\
\hline $\begin{array}{l}\text { Material für } \\
\text { Schüler*innen }\end{array}$ & $\begin{array}{l}\text { Förder- (z. B. Lesestreifen mit Lesestrategie- } \\
\text { Instruktionen) und Reflexionstools, u. v.m. (Cor- } \\
\text { nelsen Verlaga) }\end{array}$ & $\begin{array}{l}\text { Förder- (Kartenset mit Lese- } \\
\text { strategie-Instruktionen) und } \\
\text { Reflexionstool }\end{array}$ \\
\hline $\begin{array}{l}\text { Material für } \\
\text { Lehrer*innen }\end{array}$ & $\begin{array}{l}\text { Diagnosetool (3-teiliger Fahrplan), Projektma- } \\
\text { nagement-Ordner }\end{array}$ & $\begin{array}{l}\text { Diagnosetool, Einführungsse- } \\
\text { quenz für Lesestrategiefächer }\end{array}$ \\
\hline Entwicklung & $\begin{array}{l}\text { Bildungspolitisch initiiert durch das Institut für } \\
\text { Qualitätsentwicklung an Schulen Schleswig- } \\
\text { Holstein (IQSH) }\end{array}$ & $\begin{array}{l}\text { Einzelmaßnahmen durch Lehr- } \\
\text { kräfte }\end{array}$ \\
\hline Organisation & $\begin{array}{l}1 \text { geschulter Lesecoach pro Schule, ursprünglich } \\
\text { mit Entlastungsstunden }\end{array}$ & $\begin{array}{l}1 \text { Koordinator/-in pro Schule } \\
\text { (freiwillig, ohne Entlastung) }\end{array}$ \\
\hline Fortbildungen & Vorhanden/mitgedacht & Nicht organisiert \\
\hline
\end{tabular}

${ }^{a}$ Lesen macht stark - Materialordner (ISBN 978-3-06-060002-1 ), Lesen macht stark - Lesemappe (ISBN 978-3-06-060001-4)

Verbünde in einem Kontrollgruppendesign begleitet werden, deren inhaltliche Ziele den BiSS-Modulen „Diagnose und Förderung des Leseverständnisses“, „Lese- und Schreibstrategien im Verbund vermitteln“, „Selbstreguliertes Lesen und Schreiben“ sowie ,Sprachliche Bildung in fachlichen Kontexten“"zugeordnet waren. In dem Verbund „Niemanden zurücklassen“ (NZL) wurde in Schleswig-Holstein das Programm „Lesen macht stark“ für die Sekundarstufe eingesetzt, während in Oberfranken (OF) ein „Lesestrategiefächer“ zum Einsatz kam.

Eine interessante Konstellation zur Evaluation der Implementation evidenzbasierter Maßnahmen zur Leseförderung ergab sich zwischen diesen beiden Verbünden insofern, als zwei deutlich unterschiedliche Konzepte realisiert wurden. Im Verbund NZL wurde eine umfangreiche Materialsammlung mit Nutzungshinweisen für Lehrkräfte zur Verfügung gestellt. Die Implementation im Verbund NZL war ursprünglich bildungspolitisch initiiert und wurde durch geschulte Lesecoaches begleitet. Die Maßnahme wurde also administrativ unterstützt und top-down implementiert. Im Verbund OF wurde ein kompakter Lesestrategiefächer eingesetzt. Die Arbeit im Verbund $\mathrm{OF}$ ging auf die Initiative einzelner Lehrkräfte zurück und wurde durch das freiwillige Engagement von Schulkoordinator*innen getragen (bottom-up). Eine Gegenüberstellung dieser und weiterer charakteristischer Merkmale der beiden Verbünde ist stichpunktartig in Tab. 1 zusammengestellt.

Eine theoretische Analyse der beiden Materialsammlungen ergab zusammenfassend, dass sie mit einer Anleitung kognitiver und metakognitiver Strategien den aktuellen Forschungsstand zu einer Vermittlung selbstregulierten Lesens berücksichtigen. Es ist daher angemessen, von evidenzbasierten Konzepten zur Leseförderung zu sprechen. Ausführlichere Beschreibungen und Analysen der Materialien finden sich in Veröffentlichungen von Jost et al. (2018), Karstens et al. (2019) und Schmitz et al. (2021). 


\subsection{Implementationsmodelle für den schulischen Kontext}

In der Implementationsforschung werden häufig die Kriterien der Akzeptanz, Angemessenheit, Machbarkeit, Wiedergabetreue, Implementationskosten, Durchdringung und Nachhaltigkeit als Indikatoren für eine gelungene Implementation betrachtet (Proctor et al. 2009). Im Grunde decken diese Kriterien eine zeitliche Spanne ab, die sich von ersten Begegnungen mit einer Maßnahme (wie akzeptabel erscheint sie) über Erfahrungen mit deren Einsatz (z. B. Wiedergabetreue) bis hin zu der Entscheidung für eine routinemäßige Nutzung im schulischen Alltag erstreckt (vgl. auch Hasselhorn et al. 2014).

Ob der Transfer von Innovationen im schulischen Kontext gelingt, ist von einer Vielzahl an Bedingungen abhängig. Beschreibungen einzelner Fallbeispiele zur Einführung innovativer Maßnahmen an Schulen (z.B. Altrichter und Posch 1996) oder Analysen aus Modellversuchen (z. B. Berkemeyer et al. 2008) illustrieren dabei anschaulich das komplexe Zusammenspiel unterschiedlicher Faktoren. Diese liegen zum einen bei den zu implementierenden Maßnahmen selbst und zum anderen im schulischen Umfeld begründet (Gräsel 2010; Souvignier 2020). Eine Illustration zur Systematisierung für ein solches Bedingungsmodell ist in Abb. 1 dargestellt. Hier finden sich zunächst Merkmale der zu transferierenden Maßnahmen. Materialien können einfach oder sehr umfassend sein, sie beziehen sich auf unterschiedliche Inhalte, die Einführung kann durch Fortbildungsmaßnahmen begleitet sein (oder auch nicht). So zeigte sich beispielsweise, dass Maßnahmen, die als zu komplex und zeitaufwändig wahrgenommen wurden und die nicht in Einklang mit persönlichen Überzeugungen der Lehrkräfte standen, in geringerem Maße Akzeptanz fanden (Elliott 1988; Wickstrom und Witt 1993). Die Frage nach der Passung zwischen der Maßnahme und der konkreten schulischen Anwendungssituation leitet zu dem Komplex des schulischen Umfelds über: zu implementierende Maßnahmen sollten zu den Bedürfnissen von Schüler*innen sowie zu den Lehrkräften passen (Albin et al. 1996; Lindo und Elleman 2010). In Bezug auf das schulische Umfeld werden typischerweise die vier Aspekte der konkret zu unterrichtenden Schüler*innen (z. B. deren

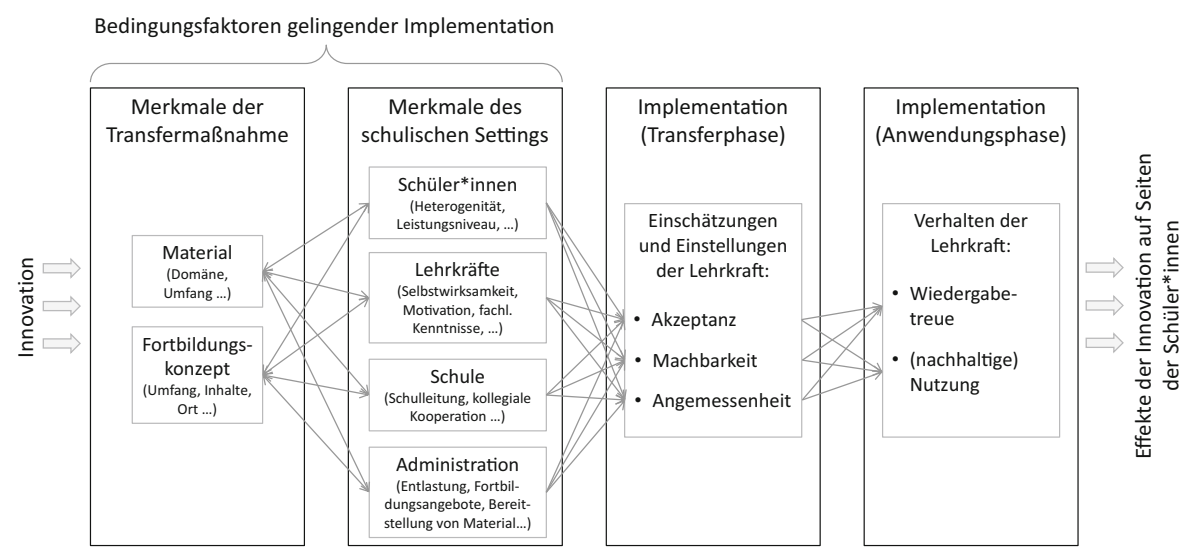

Abb. 1 Bedingungsmodell für den Transferprozess. (Vgl. Meudt 2019; Souvignier 2020) 
Heterogenität, Leistungsfähigkeit), individuelle Eigenschaften auf Seiten der Lehrkraft (Selbstwirksamkeitserleben, Motivation, Kenntnisse), Merkmale der Schule (kollegiale Kooperation, Unterstützung durch die Schulleitung), aber auch die administrative Unterstützung (Entlastung, Fortbildungsangebote) als Bedingungsfaktoren genannt (Gräsel 2010; Hasselhorn et al. 2014; Teerling und Köller 2019).

Wenn die Bedingungsfaktoren sich in einer stimmigen Weise ergänzen, dann sollte sich das in hoher Akzeptanz, hoher wahrgenommener Machbarkeit und der Einschätzung von Angemessenheit seitens der Lehrkräfte niederschlagen (vgl. Clarke und Hollingsworth 2002). Inwieweit einzelne Bedingungsfaktoren eine besonders herausgehobene Bedeutung haben oder ob eher das Zusammenspiel aus konkreten Merkmalen entscheidend ist, ist nicht eindeutig geklärt. $\mathrm{Zu}$ erwarten ist, dass günstige Bedingungen und die resultierenden Akzeptanz- und Machbarkeitseinschätzungen längerfristig zu einem entsprechenden Verhalten von Lehrkräften beitragen, beispielsweise im Hinblick auf eine hohe Implementationstreue (O'Donnell 2008) und nachhaltige Nutzung (Hasselhorn et al. 2014).

\section{Die vorliegende Studie und Fragestellungen}

In dem BiSS-Evaluationsprojekt EILe wurde die Implementation zweier Konzepte zur Leseförderung über einen Zeitraum von zwei Schuljahren begleitet. Insgesamt wurden dabei vier methodische Zugänge miteinander kombiniert: Die Materialien für die Leseförderung wurden im Hinblick auf deren theoretische Fundierung analysiert, es wurden Unterrichtsbeobachtungen durchgeführt, die Lehrkräfte wurden befragt und die Leistungsentwicklung der Schüler*innen wurde im Rahmen eines Kontrollgruppendesigns mit standardisierten Testverfahren erhoben. Die Analyse der Förderkonzepte zeigte, dass in beiden Konzepten zentrale Aspekte zur Förderung selbstregulierten Lesens berücksichtigt wurden und sie sich vorwiegend auf das Fach Deutsch beziehen (s. oben). Die Ergebnisse aus den Unterrichtsbeobachtungen legen allerdings nahe, dass die Materialien in den Verbünden eher selten zum Einsatz kamen (Schmitz et al. 2021). Während die Förderansätze im Deutschunterricht sporadisch genutzt wurden, wurde im Fachunterricht kaum darauf zurückgegriffen. Prinzipien strategieorientierter Leseförderung (u. a. Einsatz kognitiver und metakognitiver Lesestrategien, explizite Strategieinstruktion) kamen in den beiden Verbünden im Rahmen der beobachteten Unterrichtsstunden in vergleichbar geringem Umfang zum Einsatz. Erste Auswertungen der Befragung der Lehrkräfte, in denen ebenfalls von einer zurückhaltenden Nutzung der Materialien berichtet wird, stehen in Einklang mit diesen Beobachtungsdaten (Schmitz et al. 2021). Dieses Befundmuster zeigte sich weitgehend übereinstimmend für die beiden Verbünde und unabhängig von den unterschiedlichen Konzepten, die jeweils realisiert wurden. Ziel des vorliegenden Beitrags ist es, die beiden fortgeschrittenen Befragungs- und Testzeitpunkte des Evaluationsprojekts am Ende des ersten und des zweiten Projektschuljahres in den Blick zu nehmen. Dies erscheint vor allem vor dem Hintergrund der zeitlichen Erstreckung von Implementationsprozessen interessant (Hasselhorn et al. 2014). 
Um Hinweise zum Implementationsprozess aus der Perspektive der Lehrkräfte zu analysieren, werden drei Fragestellungen zu jeweils zwei Befragungszeitpunkten behandelt. Konkret werden dazu Variablen auf der Ebene der Lehrkräfte selbst (Einstellungen zu Leseförderung, Motivation bzgl. Leseförderung, Selbstwirksamkeit bzgl. Leseförderung und Wissen über Leseförderung), der Schule (Kooperation innerhalb der und zwischen den Schulen), sowie bezogen auf Einschätzungen und Einstellungen zum Fördermaterial (Akzeptanz, Machbarkeit) und das Verhalten der Lehrkräfte (Wiedergabetreue, Nutzung) herangezogen.

Zunächst wird ein Vergleich zwischen den beiden Verbünden hinsichtlich ausgewählter Variablen des Bedingungsmodells angestellt. Erwartet wird, dass sich die Implementationsbedingungen auf der Ebene der Lehrkräfte selbst und der Schule zwischen den beiden Verbünden nicht unterscheiden. Aufgrund der Unterschiedlichkeit der Maßnahmen, die sich zum einen auf die Implementationsstrategie (topdown vs. bottom-up) und zum anderen auf Umfang und Inhalte der Förderkonzepte bezieht, gehen wir davon aus, dass sich hinsichtlich der resultierenden Implementationsmerkmale der Akzeptanz, der Machbarkeit, der Wiedergabetreue und der Nutzung Unterschiede zwischen den Bedingungen ergeben.

Ergänzend wird geprüft, ob Unterschiede zwischen den Angaben von Deutschund Fachlehrkräften vorliegen. Da eine strategieorientierte Leseförderung im Fachunterricht bislang noch eher wenig verbreitet ist und sich die Bedeutung/der Stellenwert von Leseförderung im Dienste des fachlichen Lernens vom Deutschunterricht unterscheidet (Schmellentin 2018) sowie das Lesefördermaterial überwiegend auf das Fach Deutsch ausgerichtet war, wird erwartet, dass Unterschiede sowohl im Hinblick auf die Bedingungsfaktoren (Einstellungen, Selbstwirksamkeit, Wissen zu Leseförderung und kollegiale Kooperation) als auch auf die Implementationsmerkmale vorliegen.

In einem dritten Schritt werden Zusammenhänge zwischen den Bedingungsfaktoren und den Implementationsmerkmalen ausgewertet, um zu prüfen, ob einzelne Variablen sich als zentrale Bedingungen für eine gelingende Implementation erweisen oder ob eher davon auszugehen ist, dass die gesamte Breite der in den Implementationsmodellen aufgeführten Merkmale sich als bedeutsam erweist (vgl. Abschnitt 2.2). Dabei gehen wir davon aus, dass generell alle in den Implementationsmodellen aufgeführten Merkmale zum Gelingen von Implementationsprozessen beitragen. In diesem Sinne erwarten wir Interkorrelationsmuster mit durchgängig hoher Ausprägung der wechselseitigen Zusammenhänge.

Als weiteres zentrales Kriterium für eine wirksame Implementation wurden Effekte hinsichtlich der Veränderung von Lesekompetenzen bei den Schüler*innen untersucht. Als zentrale Merkmale wurden hierzu das Leseverständnis, die Leseflüssigkeit, das Lesestrategiewissen und die Lesefreude der Lernenden einbezogen, die auch durch die Förderkonzepte in den BiSS-Verbünden adressiert werden sollten.

Es wird erwartet, dass die Lesekompetenzen in den BiSS-Verbünden sich positiver entwickeln als in der Kontrollgruppe, in der keine spezifische Leseförderung implementiert wurde. Da sich im Rahmen der Unterrichtsbeobachtungen zeigte, dass das jeweilige Fördermaterial in unterschiedlicher Intensität genutzt wurde, wurde zusätzlich explorativ ausgewertet, ob sich Klassen mit hohem im Vergleich zu gerin- 
gem Materialeinsatz hinsichtlich der Veränderung der Lesekompetenz voneinander unterscheiden.

\section{Methode}

\subsection{Untersuchungsdesign und Stichprobe: Lehrkräftebefragungen}

Zur Beantwortung der Fragestellungen 1 bis 3 wurden Lehrkräftebefragungen mit standardisierten Instruktionen in den BiSS-Verbünden durchgeführt, die neben Deutschlehrkräften auch Fachlehrkräfte (in NZL Mathematik, in OF Biologie) einbezogen. Die hier verwendeten Daten stellen einen Ausschnitt aus den Gesamtbefragungen dar. Wegen hoher Dropout-Raten und wechselnder Beteiligung von Lehrkräften an den Befragungen war eine zuvor geplante längsschnittliche Betrachtung nicht möglich. Da die Beteiligung an der Online-Befragung sehr gering ausfiel, wurden die Fragebögen als Papierversion an die Lehrkräfte versandt und mit Hilfe der Verbundkoordinator*innen gebündelt an das Projektteam zurückgeschickt. Der Rücklauf war dennoch auch in der Paper-Pencil-Version vergleichsweise gering. Da die Befragungen sich auf eine große Zahl potenziell für die Implementation relevanter Variablen wie Eigenschaften der Lehrkräfte, Schulen und Materialien bezogen, wurden einige Skalen nur zu einzelnen Zeitpunkten vorgegeben, während andere Variablen wiederholt abgefragt wurden. Auf diese Weise sollte der zeitliche Umfang der Befragung so gering wie möglich gehalten werden. Die Befragung bestand im Wesentlichen aus geschlossenen Items zu Themenbereichen wie Förderpraxis, Einschätzung des Implementationsprozesses, persönlichen Eigenschaften wie Einstellungen. Die Beantwortung erfolgte im Likert-Format (sieben- bis achtstufige Skalen) oder als Ja-Nein-Antwort. Wurde mehr als die Hälfte der Items einer Skala nicht beantwortet, wurde die entsprechende Skala als fehlend behandelt. Tab. 2 gibt einen Überblick über die hier einbezogenen Skalen mit ihren Merkmalen, Quellen und Messzeitpunkten.

Die aus den Lehrkräftebefragungen ausgewählten Variablen stammen aus den Befragungen am Ende des ersten Projektschuljahres (MZP 2: Frühjahr/Sommer 2017) und Ende des zweiten Projektschuljahres (MZP 3: Frühjahr/Sommer 2018) und erlauben einen Blick auf die Rahmenbedingungen und Konsequenzen zu einem fortgeschrittenen Zeitpunkt des Projekts und des Implementationsprozesses.

Der Rücklauf lag zum zweiten Messzeitpunkt bei Abgaben von 100 Lehrkräften (Rücklaufquote $=33 \%$ ) und zum dritten Messzeitpunkt bei 98 Lehrkräften (Rücklaufquote $=32 \%$ ). Nicht von allen Lehrkräften liegen demographische Angaben vor, da die Angaben freiwillig waren.

Zum zweiten Messzeitpunkt waren $57 \%$ der Befragten Deutschlehrkräfte, $78 \%$ der Befragten waren weiblich mit einem mittleren Alter von 40,78 Jahren $(S D=10,23$ Jahre; Range 25-63), die im Mittel über eine Berufserfahrung von 14,47 Jahren ( $S D=10,86$ Jahre; Range 0-42) verfügten. 23,5\% unterrichteten an einer Realschule, 3,7\% an einem Gymnasium und 53,1\% an einer Schule mit mehreren Bildungsgängen. Zum dritten Messzeitpunkt waren 66\% der Befragten Deutschlehrkräfte, $77 \%$ der Befragten waren weiblich mit einem mittleren Alter 


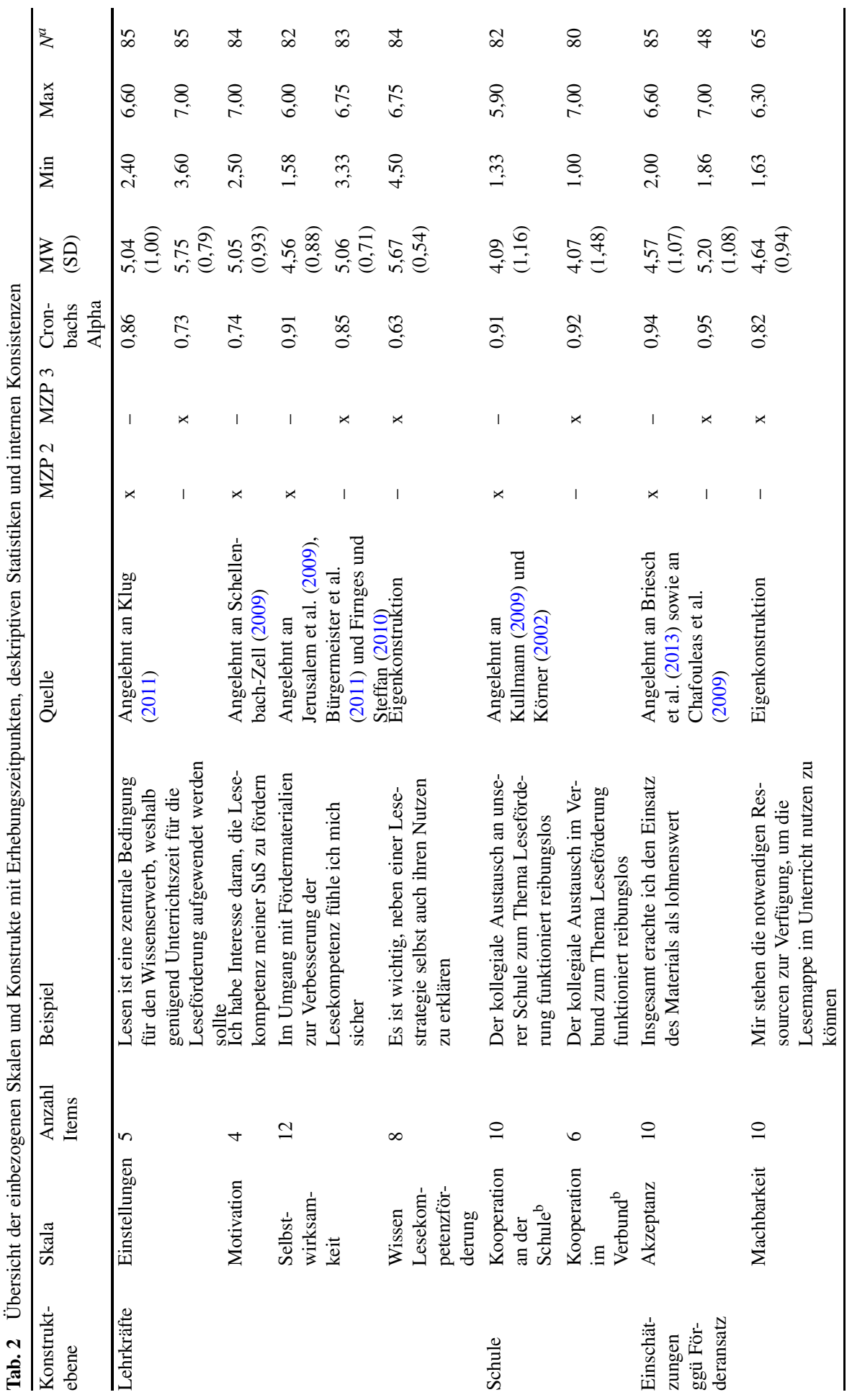




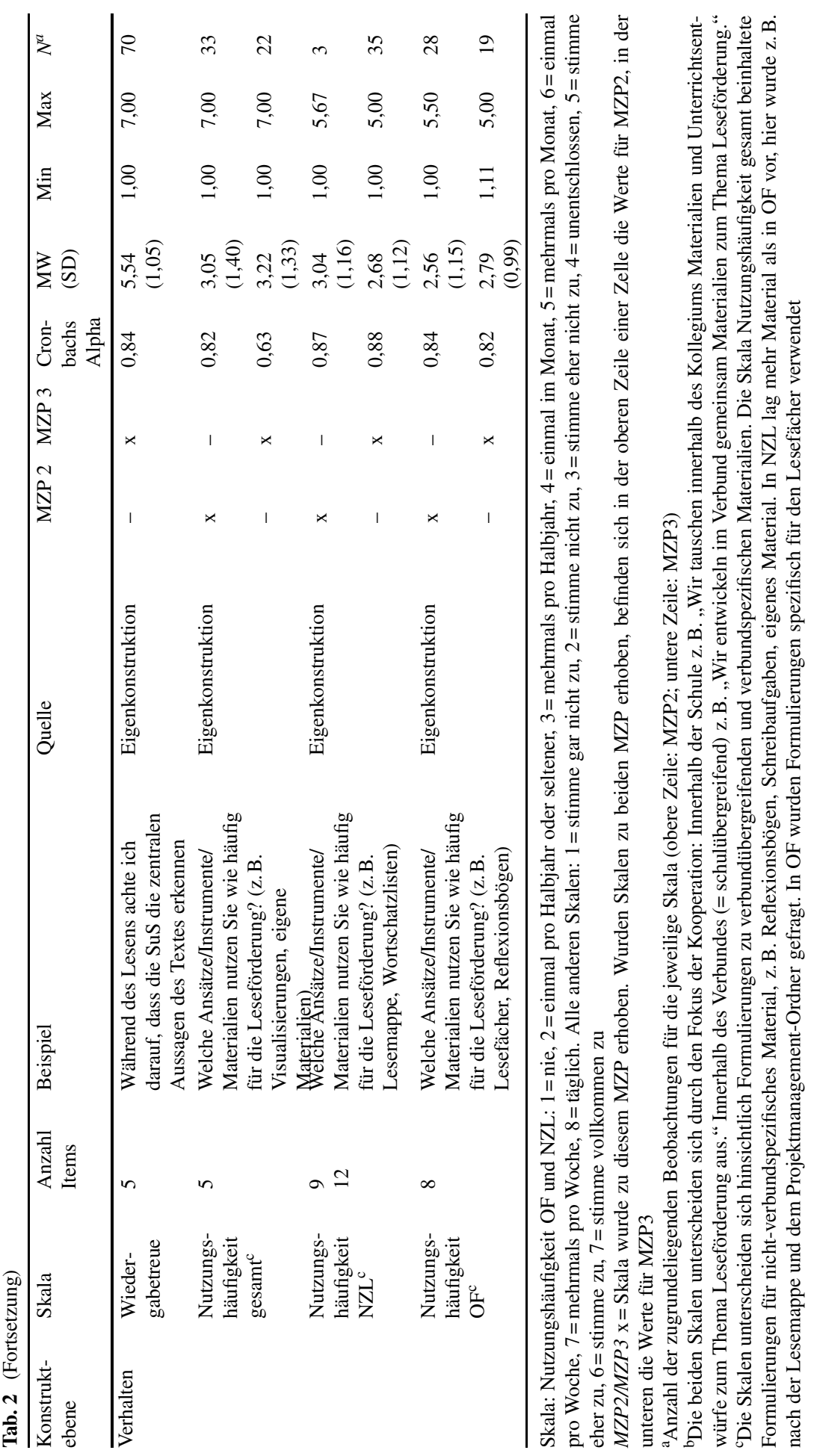


von 43,65 Jahren ( $S D=10,66$ Jahre; Range 27-65), die im Mittel über eine Berufserfahrung von 16,72 Jahren $(S D=10,97$ Jahre; Range 0,5-42) verfügten. Die Schulform wurde zum dritten Zeitpunkt nicht erhoben.

\subsection{Untersuchungsdesign und Stichprobe: Kompetenztestungen der Schüler*innen}

Zur Beantwortung der Frage 4 wurden Daten aus den Kompetenztestungen der Schüler*innen ausgewählt. Die Kompetenztestungen waren als Längsschnitt über die beiden Projektschuljahre angelegt und fanden dreimal statt. Es wurde ein Kontrollgruppendesign mit Messwiederholung realisiert, bei dem neben den Schulen der beiden Verbünde Kontrollschulen aus Nordrhein-Westfalen teilnahmen, an denen keine spezifischen Lesefördermaßnahmen umgesetzt wurden. Die Testungen wurden durch geschulte studentische Mitarbeiter*innen des Projektteams unter weitestgehend standardisierten Bedingungen durchgeführt und umfassten jeweils etwa zwei Schulstunden. Dabei wurden neben klassischen Kompetenzfacetten wie Leseflüssigkeit, Leseverständnis und Lesestrategiewissen auch Einstellungen und Wahrnehmungen des Unterrichts auf Seiten der Schüler*innen erfasst. Tab. 3 zeigt die im

Tab. 3 Überblick über die in den Kompetenztestungen erfassten Konstrukte und ihre Kennzeichen

\begin{tabular}{|c|c|c|c|c|c|}
\hline Konstrukt & Verfahren & Itemanzahl & $\begin{array}{l}\text { Theoretischer } \\
\text { Range }\end{array}$ & Reliabilität & $N^{\mathrm{a}}$ \\
\hline $\begin{array}{l}\text { Lese- } \\
\text { flüssigkeit }\end{array}$ & $\begin{array}{l}\text { Salzburger Lesescreening } \\
\text { (SLS 2-9; Wimmer und } \\
\text { Mayringer 2014) }\end{array}$ & $\begin{array}{l}100 \\
\text { (Speed- } \\
\text { test) }\end{array}$ & $\begin{array}{l}\text { Summenscore } \\
0-100\end{array}$ & $\begin{array}{l}\text { Paralleltest- } \\
\text { korrelation: } \\
0,87-0,95^{\mathrm{b}}\end{array}$ & - \\
\hline \multirow[t]{3}{*}{$\begin{array}{l}\text { Lese- } \\
\text { verständnis }\end{array}$} & \multirow{3}{*}{$\begin{array}{l}\text { Subskala Sachtext des } \\
\text { Frankfurter } \\
\text { Leseverständnistests } \\
\text { (FLVT 5-6; Souvignier } \\
\text { et al. 2008) }\end{array}$} & \multirow[t]{3}{*}{18} & \multirow[t]{3}{*}{$\begin{array}{l}\text { Summenscore } \\
0-18\end{array}$} & $\alpha=0,64(\mathrm{t} 1)$ & $\begin{array}{l}754 \\
(\mathrm{t} 1)\end{array}$ \\
\hline & & & & $0,68(\mathrm{t} 2)$ & $\begin{array}{l}755 \\
(\mathrm{t} 2)\end{array}$ \\
\hline & & & & $0,65(\mathrm{t} 3)$ & $\begin{array}{l}707 \\
(\mathrm{t} 3)\end{array}$ \\
\hline \multirow{3}{*}{$\begin{array}{l}\text { Lese- } \\
\text { strategie- } \\
\text { wissen }\end{array}$} & \multirow{3}{*}{$\begin{array}{l}\text { Items aus dem } \\
\text { Würzburger } \\
\text { Lesestrategie-Wissenstest } \\
\text { (WLST 7-12; } \\
\text { Schlagmüller und } \\
\text { Schneider 2007) }\end{array}$} & \multirow{3}{*}{$\begin{array}{l}\text { Vier } \\
\text { Aufgaben } \\
\text { à fünf bis } \\
\text { sieben Al- } \\
\text { ternativen }\end{array}$} & \multirow{3}{*}{$\begin{array}{l}\text { Summenscore } \\
0-62\end{array}$} & $\alpha=0,76(\mathrm{t} 1)$ & 851 \\
\hline & & & & & $(\mathrm{t} 1)$ \\
\hline & & & & $0,79(\mathrm{t} 2, \mathrm{t} 3)$ & $\begin{array}{l}838 \\
(\mathrm{t} 2) \\
858 \\
(\mathrm{t} 3)\end{array}$ \\
\hline \multirow[t]{3}{*}{ Lesefreude } & \multirow{3}{*}{$\begin{array}{l}\text { Adaptierte Items aus } \\
\text { PISA (Klieme et al. } \\
\text { 2010) }\end{array}$} & \multirow[t]{3}{*}{11} & \multirow[t]{3}{*}{$\begin{array}{l}\text { Mittelwert } \\
1-4\end{array}$} & $\alpha=0,84(\mathrm{t} 1)$ & $\begin{array}{l}893 \\
(\mathrm{t} 1)\end{array}$ \\
\hline & & & & $0,87(\mathrm{t} 2)$ & $\begin{array}{l}896 \\
(t 2)\end{array}$ \\
\hline & & & & $0,89(\mathrm{t} 3)$ & $\begin{array}{l}892 \\
(\mathrm{t} 3)\end{array}$ \\
\hline Kontrollvariable & Wortschatztest des CFT- & 30 & Summenscore & $\alpha=0,75$ & 452 \\
\hline Wortschatz & 20R (Weiss 2006) & & $0-30$ & & $(\mathrm{t} 1)$ \\
\hline
\end{tabular}

${ }^{a} A n z a h l$ der zugrundeliegenden Beobachtungen für die jeweilige Skala. $\alpha=$ Cronbachs Alpha. $\mathrm{t} 1=$ erster Messzeitpunkt, t2 = zweiter Messzeitpunkt, t3 = dritter Messzeitpunkt. Leseflüssigkeit, Leseverständnis, Lesestrategiewissen und Lesefreude wurden zu beiden Messzeitpunkten erhoben, Wortschatz nur zu t1

bAngaben aus dem Testmanual 
vorliegenden Beitrag einbezogenen Skalen und ihre Merkmale sowie die Quellen der Instrumente.

Es erfolgte eine Längsschnitterhebung mit insgesamt 1054 Schüler*innen, von denen 814 an allen drei Messzeitpunkten teilnahmen (im Mittel 10,32 Jahre alt, $S D=0,57$ Jahre, Range $=9-13$ Jahre). Davon stammten $22 \%$ aus NZL, $49 \%$ aus OF und $29 \%$ aus der KG. $50 \%$ waren weiblich, $78 \%$ gaben als Muttersprache Deutsch an. Als Kontrollvariablen wurden neben dem Geschlecht und der Muttersprache der Wortschatz $(M=18,69, S D=4,57)$ sowie als Indikator für den sozioökonomischen Status der Buchbesitz $(M=3,64, S D=1,16$; Paulus 2009) herangezogen. Zusätzlich wurde wegen der unterschiedlich über die Verbünde verteilten Schulformen berücksichtigt, ob es sich um Gymnasien oder Gesamt-/Realschulen handelte. Für die explorative Fragestellung nach unterschiedlichen Effekten in Klassen mit und ohne Materialeinsatz wurden auf Basis der Unterrichtsbeobachtungen aus dem zweiten Projektjahr zwei Gruppen von Klassen gebildet, in denen Materialeinsatz wie intendiert (d.h. Nutzung des über den Verbund bereitgestellten Materials) stattgefunden hatte bzw. in denen das Material nicht (wie intendiert) eingesetzt wurde. Diese beiden Gruppen wurden wiederum hinsichtlich der Entwicklung der Lesekompetenz miteinander verglichen.

Die Gesamtdatensätze des Evaluationsprojekts und eine entsprechende Dokumentation liegen unter der folgenden Quelle vor: Souvignier et al. (2020).

\subsection{Datenanalysen}

Alle Analysen erfolgen mit der SPSS Version 26 (IBM Corp. 2019) bzw. für die Entwicklung der Schüler*innenkompetenzen mit Mplus 6.1 (Muthén und Muthén 2010).

Fragestellungen 1 und 2 (Unterschiede auf Ebene der Lehrkräfte zwischen den Verbünden bzw. zwischen Deutsch- und Fachlehrkräften) werden varianzanalytisch geprüft (Post-Hoc-Analysen mit Bonferroni-Korrektur; genaugenommen wurden mehrere t-Tests unter der Annahme von Varianzgleichheit durchgeführt, da in die Varianzanalyse nur zwei Gruppen (NZL und OF) aufgenommen wurden). Die dritte Fragestellung (Zusammenhänge zwischen den Bedingungsfaktoren und den Implementationsmerkmalen) wird anhand von Korrelationen beantwortet. Dabei ergeben sich zusätzlich zu den Korrelationsmustern in der Gesamtstichprobe insgesamt vier weitere mögliche Muster (zwei Verbünde×zwei Messzeitpunkte). Im vorliegenden Beitrag werden nur die Ergebnisse für die gesamte Stichprobe zu den beiden Zeitpunkten berichtet. Die Ergebnisse getrennt nach Verbünden finden sich im OnlineSupplement. Zur Betrachtung von Frage 4 (Entwicklung der Lesekompetenz der Schüler*innen) werden latente Wachstumskurvenmodelle (latent growth curve models, LGCM; Bollen und Curran 2006) mit Regression der slopes auf die Verbundzugehörigkeit unter Kontrolle der herangezogenen Variablen Wortschatz, Geschlecht, Muttersprachlichkeit, Buchbesitz und Schulform und unter Berücksichtigung der Mehrebenenstruktur der Daten (Schüler*innen genested in Schulklassen; vgl. Cohen et al. 2003) betrachtet. Fehlende Werte werden mit Hilfe von Full Information Maximum Likelihood (FIML; Graham 2009) behandelt. 


\section{Ergebnisse}

\subsection{Ergebnisse der Lehrkräftebefragungen (Fragestellungen 1 bis 3)}

Bezüglich der ersten Fragestellung zu Unterschieden zwischen den beiden Verbünden hinsichtlich der Bedingungsfaktoren und Implementationsvariablen zeigte sich zum Ende des ersten Projektjahres, dass innerhalb einer Schule häufiger in NZL als in OF kooperiert wurde und dass ein höheres Maß an Materialnutzung im Verbund NZL angegeben wurde. Hinsichtlich der Akzeptanz auf Seiten der Lehrkräfte liegen keine Unterschiede zwischen den Verbünden vor. Zum dritten Messzeitpunkt fällt die Selbstwirksamkeit in OF höher aus als in NZL. Die Implementationstreue ist am Ende des zweiten Projektjahres ebenfalls im Verbund OF höher. Die Ergebnisse sind in Tab. 4 dargestellt. Aufgrund der zu geringen Überschneidung der beiden Lehrkräfte-Stichproben zwischen den beiden Messzeitpunkten ist ein direkter Vergleich im Sinne eines Trends über die Zeit hinweg leider nicht möglich.

Die Ergebnisse zu der zweiten Fragestellung sind in Tab. 5 aufgeführt. Erwartungskonform zeigen sich hier Unterschiede zu Gunsten der Deutschlehrkräfte in allen Skalen zum zweiten und in mehreren Skalen zum dritten Messzeitpunkt. Entgegen der Erwartungen weisen aber Fachlehrkräfte zum dritten Messzeitpunkt keine ungünstigeren förderbezogenen Einstellungen, keine geringere Kooperation auf Verbundebene, keine geringere Akzeptanz oder Einschätzung der Machbarkeit und auch keine geringere Nutzungshäufigkeit (Ausnahme: NZL-spezifisches Material) auf als Deutschlehrkräfte.

Tab. 4 Unterschiede in den ausgewählten Variablen aus der Befragung der Lehrkräfte zwischen den Verbünden zum zweiten und zum dritten Messzeitpunkt

\begin{tabular}{llll}
\hline Skala & NZL & OF & Signifikante Unterschiede \\
& $M(S D)$ & $M(S D)$ & \\
\hline Messzeitpunkt 2 & & \\
Einstellungen & $5,06(0,95)$ & $5,03(1,06)$ & $n . s$. \\
Motivation & $5,08(0,85)$ & $5,02(1,00)$ & $n . s$. \\
Selbstwirksamkeit & $4,67(0,84)$ & $4,45(0,92)$ & $n . s$. \\
Kooperation an der Schule & $4,46(1,02)$ & $3,77(1,18)$ & $F(1,89)=8,72 ; p<0,01$ \\
Akzeptanz & $4,69(1,05)$ & $4,47(1,08)$ & $n . s$. \\
Nutzungshäufigkeit gesamt & $3,46(1,51)$ & $2,68(1,18)$ & $F(1,93)=7,94 ; p<0,01$ \\
Messzeitpunkt 3 & & & \\
Einstellungen & $5,73(0,81)$ & $5,79(0,73)$ & $n . s$. \\
Selbstwirksamkeit & $4,91(0,73)$ & $5,26(0,65)$ & $F(1,80)=4,88 ; p<0,05$ \\
Wissen Lesekompetenzförderung & $5,62(0,59)$ & $5,73(0,48)$ & $n . s$. \\
Kooperation im Verbund & $4,13(1,43)$ & $3,93(1,61)$ & $n . s$. \\
Akzeptanz & $5,13(0,88)$ & $5,25(1,21)$ & $n . s$. \\
Machbarkeit & $4,53(0,86)$ & $4,79(1,04)$ & $n . s$. \\
Implementationstreue & $5,29(1,03)$ & $5,83(1,08)$ & $F(1,64)=4,44 ; p<0,05$ \\
Nutzungshäufigkeit gesamt & $3,36(1,47)$ & $3,02(1,15)$ & $n . s$. \\
\hline
\end{tabular}

${ }^{\mathrm{a}}$ Genaugenommen wurden mehrere t-Tests unter der Annahme von Varianzgleichheit durchgeführt, da in die Varianzanalyse nur zwei Gruppen (NZL und OF) aufgenommen wurden 
Tab. 5 Unterschiede in den ausgewählten Variablen aus der Befragung der Lehrkräfte zwischen Deutschund Fachlehrkräften zum zweiten und zum dritten Messzeitpunkt

\begin{tabular}{|c|c|c|c|}
\hline Skala & $\begin{array}{l}\text { Deutschlehrkräfte } \\
M(S D)\end{array}$ & $\begin{array}{l}\text { Fachlehrkräfte } \\
M(S D)\end{array}$ & Signifikante Unterschiede ${ }^{a}$ \\
\hline \multicolumn{4}{|l|}{$\overline{\text { Messzeitpunkt } 2}$} \\
\hline Einstellungen & $5,30(0,94)$ & $4,56(0,93)$ & $F(1,84)=12,29 ; p<0,01$ \\
\hline Motivation & $5,31(0,91)$ & $4,57(0,78)$ & $F(1,87)=15,06 ; p<0,01$ \\
\hline Selbstwirksamkeit & $4,83(0,79)$ & $4,11(0,86)$ & $F(1,92)=16,83 ; p<0,01$ \\
\hline Kooperation an der Schule & $4,33(1,09)$ & $3,72(1,18)$ & $F(1,89)=6,42 ; p<0,05$ \\
\hline Akzeptanz & $4,90(0,91)$ & $4,02(1,10)$ & $F(1,89)=16,82 ; p<0,01$ \\
\hline Nutzungshäufigkeit gesamt & $3,41(1,38)$ & $2,55(1,28)$ & $F(1,93)=9,44 ; p<0,01$ \\
\hline Nutzungshäufigkeit NZL & $3,48(0,99)$ & $2,33(1,06)$ & $F(1,45)=14,20 ; p<0,01$ \\
\hline Nutzungshäufigkeit OF & $2,91(1,22)$ & $2,04(0,78)$ & $F(1,48)=7,87 ; p<0,01$ \\
\hline \multicolumn{4}{|l|}{ Messzeitpunkt 3} \\
\hline Einstellungen & $5,89(0,68)$ & $5,42(0,92)$ & n.s. \\
\hline Selbstwirksamkeit & $5,30(0,52)$ & $4,50(0,77)$ & $F(1,84)=31,81 ; p<0,01$ \\
\hline $\begin{array}{l}\text { Wissen Lesekompetenz- } \\
\text { förderung }\end{array}$ & $5,72(0,49)$ & $5,57(0,61)$ & $F(1,83)=7,13 ; p<0,01$ \\
\hline Kooperation im Verbund & $4,16(1,61)$ & $3,90(1,18)$ & n.s. \\
\hline Akzeptanz & $5,26(1,09)$ & $4,73(1,04)$ & n.s. \\
\hline Machbarkeit & $4,75(1,00)$ & $4,39(0,76)$ & n.s. \\
\hline Implementationstreue & $5,77(0,93)$ & $4,98(1,15)$ & $F(1,68)=8,98 ; p<0,01$ \\
\hline Nutzungshäufigkeit gesamt & $3,26(1,15)$ & $3,13(1,65)$ & n.s. \\
\hline Nutzungshäufigkeit NZL & $3,05(1,12)$ & $2,21(0,95)$ & $F(1,43)=7,16 ; p<0,05$ \\
\hline Nutzungshäufigkeit OF & $2,92(0,94)$ & $2,20(1,05)$ & n.s. \\
\hline
\end{tabular}

${ }^{\mathrm{a}}$ Genaugenommen wurden mehrere t-Tests unter der Annahme von Varianzgleichheit durchgeführt, da in die Varianzanalyse nur zwei Gruppen (NZL und OF) aufgenommen wurden

Im Hinblick auf die Zusammenhänge zwischen den Bedingungsfaktoren und den Implementationsmerkmalen (Fragestellung 3) liegen am Ende des ersten Projektschuljahres wie erwartet moderate bis hohe signifikante positive Korrelationen zwischen allen betrachteten Skalen vor. Dies gilt gleichermaßen für die Gesamtstichprobe wie für die getrennte Betrachtung der beiden Verbünde. In Tab. 6 sind die Interkorrelationsmuster für diesen Messzeitpunkt abgebildet. Zu Messzeitpunkt 3 (Tab. 7) fallen die Korrelationen insgesamt etwas geringer aus, und getrennt nach Verbünden ergibt sich ein differenzierteres Bild. Die Wissensskala weist insgesamt tendenziell niedrigere Zusammenhänge zu den weiteren Variablen auf. Die Kooperation im Verbund steht nicht in nennenswertem Zusammenhang mit der Implementationstreue und der Nutzung der Förderkonzepte. Während die Akzeptanz im Verbund NZL weniger systematische Zusammenhänge mit den weiteren Variablen aufweist, ist es im Verbund OF die Selbstwirksamkeit, die sich im Interkorrelationsmuster als etwas unabhängiger erweist. Mit aller Vorsicht deutet sich hier über die Zeit hinweg an, dass nach einem Jahr alle einbezogenen Variablen von Bedeutung für eine gelingende Implementation sind, während nach zwei Projektjahren einzelne kompensatorische Muster (Möglichkeit des Ausgleichs einer geringeren Variablenausprägung durch höhere Werte in einer anderen Variablen) sichtbar werden. 
Tab. 6 Interkorrelationsmuster zwischen Bedingungsfaktoren und Implementationsmerkmalen zum zweiten Messzeitpunkt

\begin{tabular}{|c|c|c|c|c|c|}
\hline & MOT & SW & KOOPS & $\mathrm{AKZ}$ & $\begin{array}{l}\text { Nutzung } \\
\text { gesamt }\end{array}$ \\
\hline $\begin{array}{l}\text { Einstellungen } \\
\text { (EIN) }\end{array}$ & $\begin{array}{l}0,70 * * \\
0,62 * * / 0,76^{* *}\end{array}$ & $\begin{array}{l}0,79 * * \\
0,74 * * / 0,83 * *\end{array}$ & $\begin{array}{l}0,57 * * \\
0,53 * * / 0,63 * *\end{array}$ & $\begin{array}{l}0,58 * * \\
0,69 * * / 0,50 * *\end{array}$ & $\begin{array}{l}0,44 * * \\
0,48 * * / 0,46 * *\end{array}$ \\
\hline $\begin{array}{l}\text { Motivation } \\
\text { (MOT) }\end{array}$ & - & $\begin{array}{l}0,70 * * \\
0,59 * * / 0,78 * *\end{array}$ & $\begin{array}{l}0,55^{* *} \\
0,49 * * / 0,61 * *\end{array}$ & $\begin{array}{l}0,54 * * \\
0,52 * * / 0,56 * *\end{array}$ & $\begin{array}{l}0,54 * * \\
0,62 * * / 0,58 * *\end{array}$ \\
\hline $\begin{array}{l}\text { Selbstwirksamkeit } \\
\text { (SW) }\end{array}$ & - & - & $\begin{array}{l}0,73 * * \\
0,71 * * / 0,75^{* *}\end{array}$ & $\begin{array}{l}0,71 * * \\
0,81 * * / 0,62 * *\end{array}$ & $\begin{array}{l}0,46 * * \\
0,52 * * / 0,57 * *\end{array}$ \\
\hline $\begin{array}{l}\text { Kooperation } \\
\text { an der Schule } \\
\text { (KOOPS) }\end{array}$ & - & - & - & $\begin{array}{l}0,68 * * \\
0,72 * * / 0,66^{* *}\end{array}$ & $\begin{array}{l}0,43 * * \\
0,48 * * / 0,44 * *\end{array}$ \\
\hline $\begin{array}{l}\text { Akzeptanz } \\
\text { (AKZ) }\end{array}$ & - & - & - & - & $\begin{array}{l}0,42 * * \\
0,49 * * / 0,53 * *\end{array}$ \\
\hline
\end{tabular}

In der ersten Zeile ist jeweils die Korrelation in der Gesamtstichprobe vermerkt. In der zweiten Zeile steht die Korrelation für den Verbund NZL an erster Stelle und dahinter die Korrelation für den Verbund OF $* * p<0,01, * p<0,05$

In einer Hauptkomponentenanalyse wurde ergänzend geprüft, ob eine Gruppierung von Bedingungsfaktoren möglich ist. Dabei zeigte sich für beide Messzeitpunkte ein einzelner dominanter Faktor, neben dem keine bedeutsamen weiteren Faktoren identifiziert werden konnten. Dieses Ergebnis unterstützt die Annahme, dass alle in die Analysen einbezogenen Variablen Teil eines umfassenden Bedingungsgefüges sind.

\subsection{Ergebnisse der Kompetenztestungen (Fragestellung 4)}

Für die vierte Fragestellung sind die Ergebnisse zur Kompetenzentwicklung in Tab. 8 zusammengefasst. Die Ergebnisse der latenten Wachstumskurvenmodelle finden sich in Tab. 9. Insgesamt zeigt sich ein guter bis zufriedenstellender Fit für alle Modelle (vgl. Schermelleh-Engel et al. 2003). Es fällt zunächst auf, dass deskriptiv Niveauunterschiede zwischen NZL, OF und der KG vorliegen, die weitgehend über alle drei Messzeitpunkte erhalten bleiben. Bezogen auf die Entwicklung der Leseleistungen, des Lesestrategiewissens und der Lesefreude (Regression Slope auf Verbundzugehörigkeit) zeigen sich keine durchgängigen Vorteile für die beiden Verbünde im Vergleich zur KG. So fällt lediglich die Entwicklung der Leseflüssigkeit in den Verbünden OF und NZL höher aus als in der KG. Unerwartet ist, dass die Entwicklung des Lesestrategiewissens in der KG höher ausfällt als in OF. Anzumerken ist, dass die verschiedenen Kontrollvariablen unterschiedlichen Einfluss auf die Entwicklung der Kompetenzfacetten haben, während der Wortschatz und die Schulform Gymnasium durchgängig den Ausgangswert der Facetten in erwarteter Richtung beeinflussen (Gymnasialschüler*innen und Schüler*innen mit einem größeren Wortschatz erreichen höhere Ausgangswerte). Bis auf die Leseflüssigkeit gilt dies auch für den Buchbesitz (höhere Werte bedeuten höhere Ausgangswerte). Unter Kontrolle dieser Konstrukte zeigt sich nur für den Verbund OF ein höherer Ausgangswert im Leseverständnis verglichen mit der KG. 


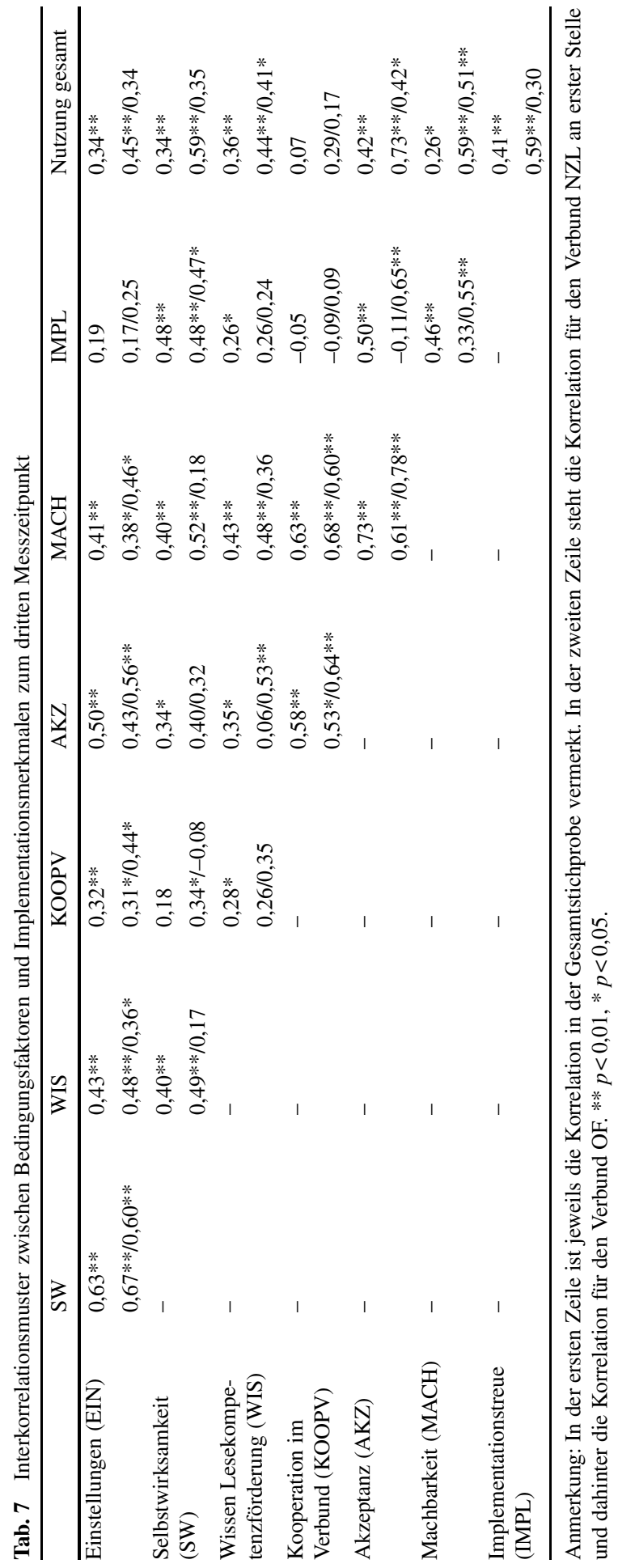


Tab. 8 Deskriptive Statistiken in den Verbünden und in der KG

\begin{tabular}{|c|c|c|c|c|c|c|}
\hline \multirow[b]{2}{*}{ Facette } & \multicolumn{2}{|l|}{ NZL } & \multicolumn{2}{|l|}{ OF } & \multicolumn{2}{|l|}{ KG } \\
\hline & $M$ & $S D$ & $M$ & $S D$ & $M$ & $S D$ \\
\hline \multicolumn{7}{|c|}{$\overline{\text { Leseflüssigkeit (SLS) }}$} \\
\hline $\mathrm{T} 1$ & 44,25 & 10,94 & 49,49 & 8,98 & 46,76 & 10,27 \\
\hline $\mathrm{T} 2$ & 49,98 & 9,96 & 56,14 & 9,53 & 51,03 & 10,17 \\
\hline $\mathrm{T} 3$ & 54,25 & 11,71 & 62,24 & 10,22 & 56,55 & 11,07 \\
\hline Differenz $\mathrm{t} 3-\mathrm{t} 1$ & 10,23 & 7,33 & 13,30 & 6,87 & 9,47 & 6,36 \\
\hline \multicolumn{7}{|c|}{ Leseverständnis (FLVT) } \\
\hline $\mathrm{T} 1$ & 10,97 & 3,36 & 13,37 & 2,60 & 11,53 & 3,47 \\
\hline $\mathrm{T} 2$ & 11,04 & 3,23 & 13,85 & 2,84 & 11,81 & 3,55 \\
\hline $\mathrm{T} 3$ & 13,18 & 4,29 & 16,50 & 4,16 & 14,32 & 4,21 \\
\hline Differenz t3-t1 & 2,51 & 3,76 & 3,20 & 3,76 & 2,55 & 3,84 \\
\hline \multicolumn{7}{|c|}{ Lesestrategiewissen (WLST) } \\
\hline $\mathrm{T} 1$ & 39,23 & 9,34 & 43,27 & 7,85 & 41,56 & 8,45 \\
\hline $\mathrm{T} 2$ & 38,71 & 8,82 & 42,87 & 8,34 & 41,79 & 8,75 \\
\hline $\mathrm{T} 3$ & 41,77 & 8,27 & 43,80 & 8,45 & 43,97 & 8,75 \\
\hline Differenz $\mathrm{t} 3-\mathrm{t} 1$ & 2,54 & 9,42 & 0,22 & 10,12 & 2,15 & 10,29 \\
\hline \multicolumn{7}{|l|}{ Lesefreude } \\
\hline $\mathrm{T} 1$ & 2,65 & 0,64 & 2,85 & 0,59 & 2,89 & 0,59 \\
\hline $\mathrm{T} 2$ & 2,62 & 0,64 & 2,80 & 0,63 & 2,72 & 0,65 \\
\hline T3 & 2,42 & 0,70 & 2,56 & 0,69 & 2,63 & 0,67 \\
\hline Differenz $\mathrm{t} 3-\mathrm{t} 1$ & $-0,22$ & 0,63 & $-0,29$ & 0,54 & $-0,27$ & 0,59 \\
\hline
\end{tabular}

T1 Erster Erhebungszeitpunkt, T2 zweiter Erhebungszeitpunkt, T3 dritter Erhebungszeitpunkt

Explorativ wird im Rahmen der vierten Fragestellung betrachtet, ob in Klassen mit Materialeinsatz (d.h. Einsatz des Verbundmaterials wie im Implementationsdesign vorgesehen) stärkere Zuwächse in den Facetten der Lesekompetenz festzustellen sind als in den Klassen, die das Verbundmaterial nicht wie intendiert oder anderes Material einsetzten (Erfassung im Rahmen der Unterrichtsbeobachtungen). Die entsprechende Gruppenbildung wurde anhand des Materialeinsatzes in den beobachteten Unterrichtsstunden vorgenommen. Die in Tab. 10 zusammengefassten Ergebnisse weisen deskriptiv etwas höhere Differenzen von t1 zu t3 in den Klassen mit höherer Materialnutzung für Leseflüssigkeit und Leseverständnis aus. In der latenten Modellierung (vgl. Tab. 9, „Einfluss von Materialeinsatz auf Slope“) zeigen sich hier aber keine statistisch bedeutsamen Unterschiede für die Entwicklung der Kompetenzfacetten unter Einbezug der Kontrollvariablen.

\section{Diskussion}

\subsection{Beantwortung der Forschungsfragen}

In der vorliegenden Studie wurde die Implementation zweier konzeptuell unterschiedlicher Maßnahmen zur Leseförderung in der Sekundarstufe miteinander ver- 
Tab. 9 Ergebnisse der latenten Wachstumskurvenmodelle zu Unterschieden in der Entwicklung von Lesekompetenzen zwischen den Gruppen

\begin{tabular}{|c|c|c|c|c|}
\hline & $\begin{array}{l}\text { Leseflüssigkeit } \\
\text { (SLS) } \\
N=952\end{array}$ & $\begin{array}{l}\text { Leseverständnis } \\
\text { (FLVT) } \\
N=952\end{array}$ & $\begin{array}{l}\text { Lesestrategiewissen } \\
\text { (WLST) } \\
N=954\end{array}$ & $\begin{array}{l}\text { Lesefreude } \\
N=954\end{array}$ \\
\hline Intercept (S.E.) & $47,12(0,59)^{* *}$ & $11,92(0,31)^{* *}$ & $41,57(0,39)^{* *}$ & $\begin{array}{l}2,82 \\
(0,03)^{* *}\end{array}$ \\
\hline Slope (S.E.) & $5,68(0,21)^{* *}$ & $1,24(0,08)^{* *}$ & $0,73(0,21)^{* *}$ & $\begin{array}{l}-0,13 \\
(0,01)^{* *}\end{array}$ \\
\hline \multicolumn{5}{|l|}{ Regression Slope auf ... } \\
\hline $\begin{array}{l}\text { Muttersprache } \\
\text { Deutsch }\end{array}$ & $-0,46(0,32)$ & $0,02(0,15)$ & $-0,01(0,43)$ & $\begin{array}{l}-0,02 \\
(0,03)\end{array}$ \\
\hline Buchbesitz & $0,10(0,11)$ & $0,13(0,05)^{* *}$ & $-0,29(0,16)$ & $\begin{array}{l}0,00 \\
(0,01)\end{array}$ \\
\hline Geschlecht & $0,83(0,23)^{* *}$ & $0,10(0,13)$ & $1,04(0,35)^{* *}$ & $\begin{array}{l}0,04 \\
(0,02)\end{array}$ \\
\hline Wortschatz & $0,04(0,03)$ & $-0,01(0,02)$ & $-0,10(0,04)^{*}$ & $\begin{array}{l}0,01 \\
(0,00)^{*}\end{array}$ \\
\hline Gymnasium & $0,84(0,34)^{*}$ & $0,32(0,18)$ & $0,64(0,50)$ & $\begin{array}{l}0,01 \\
(0,03)\end{array}$ \\
\hline OF vs. NZL & $0,87(0,48)$ & $0,06(0,22)$ & $-0,83(0,63)$ & $\begin{array}{l}-0,05 \\
(0,03)\end{array}$ \\
\hline$N Z L$ vs. $K G$ & $0,99(0,46) *$ & $0,10(0,23)$ & $-0,09(0,50)$ & $\begin{array}{l}0,04 \\
(0,04)\end{array}$ \\
\hline$O F$ vs. $K G$ & $1,87(0,37)^{* *}$ & $0,16(0,16)$ & $-0,92(0,41)^{*}$ & $\begin{array}{l}-0,02 \\
(0,03)\end{array}$ \\
\hline \multicolumn{5}{|c|}{${ }^{\text {a }}$ Regression Intercept auf ... } \\
\hline $\begin{array}{l}\text { Muttersprache } \\
\text { Deutsch }\end{array}$ & $-0,13(0,85)$ & $0,54(0,25)^{*}$ & $0,35(0,59)$ & $\begin{array}{l}-0,04 \\
(0,04)\end{array}$ \\
\hline Buchbesitz & $0,24(0,24)$ & $0,18(0,07)^{* *}$ & $0,87(0,24)^{* *}$ & $\begin{array}{l}0,14 \\
(0,02)^{* *}\end{array}$ \\
\hline Geschlecht & $2,03(0,57)^{* *}$ & $-0,17(0,15)$ & $0,92(0,53)$ & $\begin{array}{l}0,30 \\
(0,03)^{* *}\end{array}$ \\
\hline Wortschatz & $0,75(0,08)^{* *}$ & $0,29(0,03)^{* *}$ & $0,41(0,07)^{* *}$ & $\begin{array}{l}0,02 \\
(0,00)^{* *}\end{array}$ \\
\hline Gymnasium & $3,98(0,73)^{* *}$ & $1,09(0,23)^{* *}$ & $1,49(0,56)^{* *}$ & $\begin{array}{l}0,19 \\
(0,06)^{* *}\end{array}$ \\
\hline OF vs. NZL & $-0,91(0,98)$ & $0,50(0,33)$ & $0,90(0,77)$ & $\begin{array}{l}-0,09 \\
(0,07)\end{array}$ \\
\hline NZL vs. KG & $1,50(0,95)$ & $0,37(0,32)$ & $-0,53(0,68)$ & $\begin{array}{l}-0,01 \\
(0,08)\end{array}$ \\
\hline OF vs. KG & $0,59(0,78)$ & $0,87(0,22)^{* *}$ & $0,37(0,46)$ & $\begin{array}{l}-0,11 \\
(0,06)\end{array}$ \\
\hline $\begin{array}{l}\text { Einfluss von Material- } \\
\text { einsatz auf Slope }\end{array}$ & $-0,06(0,18)$ & $-0,04(0,08)$ & $-0,33(0,24)$ & $\begin{array}{l}-0,02 \\
(0,01)\end{array}$ \\
\hline
\end{tabular}


Tab. 9 (Fortsetzung)

\begin{tabular}{lllll}
\hline & $\begin{array}{l}\text { Leseflüssigkeit } \\
\text { (SLS) } \\
N=952\end{array}$ & $\begin{array}{l}\text { Leseverständnis } \\
\text { (FLVT) } \\
N=952\end{array}$ & $\begin{array}{l}\text { Lesestrategiewissen } \\
\text { (WLST) } \\
N=954\end{array}$ & $\begin{array}{l}\text { Lesefreude } \\
N=954\end{array}$ \\
\hline Fit & & & & \\
AIC & 17350,42 & 13007,08 & 18606,36 & 3686,51 \\
Adj. BIC & 17387,43 & 13044,10 & 18643,43 & 3723,57 \\
CFI & 1,00 & 0,93 & 0,97 & 0,98 \\
TLI & 1,00 & $0,78^{\mathrm{b}}$ & 0,92 & 0,94 \\
RMSEA & 0,00 & $0,13^{\mathrm{b}}$ & 0,04 & 0,06 \\
SRMR & 0,01 & 0,03 & 0,02 & 0,01 \\
\hline
\end{tabular}

S. E. Standard Error. Kodierung: Geschlecht 1 = männlich, 2 = weiblich. Verbundvariablen wurden als dummy-kodierte Variablen einbezogen

$* * p<0,01, * p<0,05$

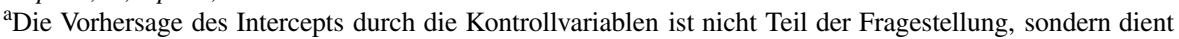
der Kontrolle von möglichen interindividuellen Unterschieden der Schüler*innen in ihren Ausgangswerten ${ }^{b}$ Die vergleichsweise hohe Abweichung zwischen TLI und CFI sowie zwischen RMSEA und SRMR ist auffällig, aber auf Basis der Daten- und Modelllage nicht eindeutig erklärbar

glichen. Zusammenfassend überwiegt bei weitem der Eindruck, dass - bei aller Unterschiedlichkeit der Transfermaßnahmen - die Implementationsmerkmale in den beiden Verbünden recht ähnlich ausfielen. Unterschiede im Ergebnismuster zeigen sich insofern, als am Ende des ersten Projektjahres die Nutzungshäufigkeit in dem administrativ stärker unterstützten Verbund NZL (Materialien werden bereitgestellt; top-down Implementationsstrategie) aus Sicht der Lehrkräfte höher ausfiel, während am Ende des zweiten Projektjahres die Implementationstreue bei dem weniger umfangreichen Material des Verbunds OF höher lag. Diese letztlich gut erklärbaren Unterschiede finden allerdings keine Entsprechungen bei den Einschätzungen der Lehrkräfte im Hinblick auf Akzeptanz oder Machbarkeit im Vergleich zwischen den Verbünden.

$\mathrm{Zu}$ beiden Messzeitpunkten zeigt sich überwiegend, dass sowohl auf der Seite der Bedingungsfaktoren als auch hinsichtlich der Implementationsmerkmale die Deutschlehrkräfte höhere Werte aufweisen als die Fachlehrkräfte. Hier spiegelt sich die Problematik einer allzu einseitigen ,Zuständigkeit“ für den Bereich der Leseförderung respektive eine unzureichende Berücksichtigung von fachlicher Ausrichtung des Materials und Involvierung der Fachlehrkräfte wider. Dabei geht es bei einer Leseförderung in den Unterrichtsfächern primär darum, dass eine fachspezifische Leseförderung auch das fachliche Lernen unterstützen kann, beispielsweise durch eine Vermittlung fachbezogener Lesestrategien, die auch die Textmusterspezifik typischer Texte in einem Fach aufgreifen (s. z. B. Schmellentin 2018). Nicht zuletzt die großen Unterschiede im Hinblick auf die Selbstwirksamkeit der Lehrkräfte weisen darauf hin, dass verstärkte empirische Forschung zu sprachlicher Bildung im Fachunterricht sowie zielgerichtete Maßnahmen zur Professionalisierung der Fachlehrkräfte wichtig wären, um hier Veränderungen bewirken zu können.

Das Zusammenhangsgefüge zwischen unterschiedlichen Bedingungsfaktoren und Implementationsmerkmalen aus Transfer- und Anwendungsphase weist am Ende des ersten Projektjahres durchgängig bedeutsame Korrelationen mit $r>0,40$ aus. 
Tab. 10 Differenzierung: Deskriptive Statistiken in Abhängigkeit des Materialeinsatzes (Verbund-Material) $\mathrm{zu} \mathrm{t} 3^{\mathrm{a}}$

\begin{tabular}{|c|c|c|c|c|}
\hline \multirow[b]{2}{*}{ Facette } & \multicolumn{2}{|c|}{$\begin{array}{l}\text { Geringer Materialeinsatz } \\
(N=826 \text { in } 42 \text { Klassen })\end{array}$} & \multicolumn{2}{|c|}{$\begin{array}{l}\text { Hoher Materialeinsatz } \\
(N=228 \text { in } 11 \text { Klassen })\end{array}$} \\
\hline & $M$ & $S D$ & $M$ & $S D$ \\
\hline \multicolumn{5}{|c|}{ Leseflüssigkeit (SLS) } \\
\hline $\mathrm{T} 1$ & 46,98 & 10,18 & 49,71 & 9,13 \\
\hline $\mathrm{T} 2$ & 52,36 & 9,98 & 57,16 & 10,09 \\
\hline $\mathrm{T} 3$ & 58,03 & 11,34 & 61,88 & 10,75 \\
\hline $\begin{array}{l}\text { Differenz t3- } \\
\text { t1 }\end{array}$ & 11,12 & 7,33 & 12,90 & 5,62 \\
\hline \multicolumn{5}{|c|}{ Leseverständnis (FLVT) } \\
\hline $\mathrm{T} 1$ & 12,06 & 3,31 & 13,37 & 2,60 \\
\hline $\mathrm{T} 2$ & 12,40 & 3,48 & 13,71 & 2,66 \\
\hline $\mathrm{T} 3$ & 14,83 & 4,38 & 16,34 & 4,34 \\
\hline $\begin{array}{l}\text { Differenz t3- } \\
\text { t1 }\end{array}$ & 2,76 & 3,76 & 3,26 & 3,89 \\
\hline \multicolumn{5}{|c|}{ Lesestrategiewissen (WLST) } \\
\hline $\mathrm{T} 1$ & 41,54 & 8,61 & 43,24 & 7,96 \\
\hline $\mathrm{T} 2$ & 41,43 & 8,72 & 42,59 & 8,59 \\
\hline $\mathrm{T} 3$ & 43,60 & 8,31 & 42,80 & 9,30 \\
\hline $\begin{array}{l}\text { Differenz t3- } \\
\text { t1 }\end{array}$ & 1,80 & 9,79 & $-0,63$ & 10,91 \\
\hline \multicolumn{5}{|l|}{ Lesefreude } \\
\hline $\mathrm{T} 1$ & 2,82 & 0,61 & 2,82 & 0,62 \\
\hline $\mathrm{T} 2$ & 2,73 & 0,64 & 2,78 & 0,62 \\
\hline $\mathrm{T} 3$ & 2,57 & 0,69 & 2,47 & 0,70 \\
\hline $\begin{array}{l}\text { Differenz t3- } \\
\text { t1 }\end{array}$ & $-0,25$ & 0,59 & $-0,34$ & 0,51 \\
\hline
\end{tabular}

T1 Erster Erhebungszeitpunkt, T2 zweiter Erhebungszeitpunkt, T3 dritter Erhebungszeitpunkt

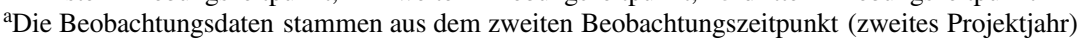

Alle erhobenen Variablen weisen substanzielle Zusammenhänge mit der Nutzung der Materialien auf. Gleichzeitig sind die Interkorrelationsmuster in der Gesamtstichprobe und den beiden einzelnen Verbünden weitestgehend vergleichbar. Das Gesamtbild hoher Interkorrelationen liegt auch am Ende des zweiten Projektjahres und bei einigen neu einbezogenen Variablen vor. An einzelnen Stellen weichen die Zusammenhänge in den beiden Verbünden numerisch voneinander $a b$, beispielsweise im Hinblick auf den Stellenwert der Akzeptanz für die Implementationstreue. Möglicherweise beeinflussen hier spezifische Rahmenbedingungen (z.B. top-down vs. bottom-up) längerfristig das Zusammenhangsgefüge der einzelnen Variablen. Auch die vereinzelten niedrigen Interkorrelationen weisen darauf hin, dass mitunter kompensatorische Bedingungen vorliegen können, sodass - längerfristig - nicht für alle potenziellen Bedingungsfaktoren gelten muss, dass diese in hohem Maße vorliegen müssen. Die insgesamt niedrigen Zusammenhänge zwischen der Kooperation im Verbund und der Wiedergabetreue sowie der Nutzung deuten darauf hin, dass diese etwas „distalere“ Form der gegenseitigen Unterstützung ein vergleichsweise 
geringeres Gewicht hat als die konkrete Kooperation innerhalb eines Kollegiums (vgl. Hebbecker et al. 2020).

Fasst man die Befunde aus dem Vergleich zwischen den Verbünden und den Zusammenhangsanalysen zusammen, so wird deutlich, dass Merkmale der Lehrkräfte sowie subjektive Einstellungen und die Wahrnehmung der Förderkonzepte sich nicht zwischen den Verbünden, wohl aber zwischen den Lehrkräften unterscheiden. Dabei zeigt sich, dass Bedingungsfaktoren und Implementationsvariablen wie Einstellung, Selbstwirksamkeit und Akzeptanz wesentliche individuelle Zusammenhänge mit der Nutzung der Tools aufweisen (vgl. Clarke und Hollingsworth 2002).

Die Befunde zu Effekten der beiden Konzepte auf der Ebene der Schüler*innen weisen nur sehr punktuell überlegene Entwicklungen gegenüber einer Kontrollbedingung ohne explizite Maßnahmen zur Leseförderung auf. In beiden Verbünden liegen mit Blick auf die Leseflüssigkeit kleine positive Effekte verglichen mit der KG vor. Gerade das Ergebnis zur Verbesserung der Leseflüssigkeit ist vor dem Hintergrund des strategieorientierten Lesefächers, der konkret auf eine Verbesserung des Leseverständnisses abzielt, zunächst überraschend. Ein Ansatz zur Erklärung dieses Ergebnisses mag in dem insgesamt recht niedrigen Niveau expliziter Lesestrategievermittlung liegen. In diesem Sinne liegt es nahe, in den Verbünden eher von einer allgemeinen Leseförderung auszugehen als von einer spezifischen strategieorientierten Förderung (vgl. Magnusson et al. 2019; Schmitz et al. 2021). Diese Annahme wird auch durch die negativen Effekte auf das Lesestrategiewissen im Verbund OF gestützt. Insgesamt lässt sich festhalten, dass die Ergebnisse für Lesekompetenzen, Lesestrategiewissen und Lesemotivation keinen überzeugenden Nachweis für die Wirksamkeit der beiden Konzepte darstellen. Die vergleichsweise geringe Nutzung der Förderkonzepte legte einen explorativen Vergleich zwischen Projektklassen mit höherer und niedrigerer Nutzung des Fördermaterials (auf der Basis von Unterrichtsbeobachtungen zu einem Messzeitpunkt) nahe. Deskriptiv zeigten sich etwas höhere Differenzwerte für Leseflüssigkeit und Leseverständnis in den Klassen, in denen die Förderkonzepte häufiger eingesetzt wurden. Diese Unterschiede erwiesen sich allerdings in der latenten Modellierung unter Einbezug der Kontrollvariablen nicht als statistisch signifikant.

Aber wie lassen sich ein durchgängig höheres $\mathrm{Ma} ß$ an Nutzung und Implementationstreue erreichen? Mit Blick auf die Befunde dieser Evaluationsstudie ist noch einmal hervorzuheben, dass trotz der großen Unterschiede zwischen den beiden Konzepten (Implementationsstrategien; Umfang des Materials) nur sehr kleine Unterschiede hinsichtlich der Bedingungsfaktoren und Implementationsmerkmale vorlagen. Es fanden sich allenfalls erste Hinweise darauf, dass Unterstützung von auBen und einfache Materialien die Nutzung und die Implementationstreue verbessern. Vielmehr weisen aber die hohen Interkorrelationen zwischen den Bedingungsfaktoren und der Nutzung darauf hin, dass die komplexen Implementationsmodelle mit ihrer Vielzahl potenziell relevanter Variablen Gültigkeit besitzen. Die Botschaft hinter diesem Befund ist herausfordernd: Es sind viel weniger einzelne herausgehobene Merkmale, die über das Gelingen von Implementationsmaßnahmen entscheiden, als vielmehr das stimmige Gesamtpaket aus Material, Unterstützungsmaßnahmen, schulischen Rahmenbedingungen und Merkmalen der Lehrkräfte, das letztlich zu einer nachhaltigen Nutzung führen kann (Clarke und Hollingsworth 2002; Hasselhorn 
et al. 2014). Dass hier längerfristig eine Kompensation einzelner Bedingungsfaktoren möglich erscheint, ist sicherlich eine entlastende Botschaft, die allerdings einen „langen Atem“ erfordert.

\subsection{Limitationen der Aussagekraft der vorliegenden Studie}

Die Evaluation der Implementationspraxis in den bestehenden BiSS-Verbünden mit den jeweils dort eingesetzten Materialien ermöglicht ein sehr realistisches Bild schulischen Alltags. Da die Konzepte der beiden Verbünde als fertiges „Paket“ vorlagen, ist eine differenzierte Analyse einzelner Wirkfaktoren für eine gelingende Implementation in dem vorliegenden Design jedoch nicht möglich. Zudem liegt eine Konfundierung der unterschiedlichen Bedingungen mit den Bundesländern der Verbünde vor. Einflüsse aufgrund solchermaßen unterschiedlicher Rahmenbedingungen sind nicht auszuschließen. Weiterhin fallen die Stichprobengrößen unterschiedlich groß aus (knapp 50\% der Schüler*innen stammen aus OF, die kleinste Gruppe bilden die Schüler*innen aus dem Verbund NZL). Angesichts des trotz vielfältiger Anstrengungen eher moderaten Rücklaufs ist zudem eine eingeschränkte Repräsentativität der Lehrer*innenstichprobe denkbar. Das Befundmuster weist allerdings darauf hin, dass nicht einzelne besonders herausragende Bedingungsfaktoren entscheidend sind, sondern dass letztlich das in Abb. 1 dargestellte komplexe Bedingungsgefüge als Ganzes über den Erfolg von Implementationsvorhaben entscheidet. Zur Untersuchung eines solchen Bedingungsgefüges wären Strukturgleichungsmodelle ein passenderer methodischer Zugang als einfache Interkorrelationsmatrizen. In der vorliegenden Studie gelang es jedoch leider nur bei zu wenigen Lehrkräften, sie verbindlich für eine längsschnittliche Teilnahme an den Befragungen zu gewinnen. Insofern müssen die beiden Befragungen als unabhängige Erhebungen betrachtet werden. Schlussfolgerungen über die Entwicklungsdynamik in Transferprozessen können daher anhand der vorliegenden Daten nicht gezogen werden. In diesem Sinne konnte auch ein Prädiktionsmodell, das eine zeitliche Staffelung von Bedingungsfaktoren und Implementationsvariablen zulässt, leider nicht geprüft werden. Aufgrund der hohen Interkorrelationen zwischen den einbezogenen Variablen liegt zudem eine starke Multikollinearität vor, an der einfache Regressionsmodelle scheitern. Dies wird auch durch den Nebenbefund gestützt, dass sich lediglich ein dominanter Faktor für die beteiligten Skalen identifizieren lässt.

Die Befunde der vorliegenden Studie erlauben zudem keine Aussage darüber, wie wirksam die eingesetzten Förderkonzepte sind, wenn sie konsequent und mit hoher Implementationstreue eingesetzt würden. Hier setzt die faktisch erfolgte Nutzung der Maßnahmen ihre Grenzen. Wichtig ist entsprechend für alle Schlussfolgerungen bei dieser Studie im Blick zu behalten, dass die hier vorgestellten Ergebnisse bei einem insgesamt relativ niedrigen Umsetzungsniveau der Programme erzielt wurden.

\subsection{Fazit}

Als zentrale Kriterien für eine gelungene Implementation wurden zu Beginn des Beitrags eine breite Nutzung und positive Effekte im Hinblick auf die Entwicklung von Schüler*innenleistungen genannt. Beide Ziele wurden im Rahmen der hier eva- 
luierten Verbünde nicht in der gewünschten Breite realisiert. Entsprechend rückt die Frage in den Fokus, welche Bedingungen eine höhere Nutzung und Implementationstreue positiv beeinflussen könnten. Aus den Befunden der vorliegenden Studie lässt sich ableiten, dass eine engere Kooperation zwischen Deutsch- und Fachlehrkräften für die Leseförderung hilfreich sein könnte. Es deutet sich auch an, dass möglichst einfache, aber mit Blick auf das Förderziel passgenaue, Materialien und die (externe) Unterstützung durch Fortbildungsmaßnahmen förderlich sind. Darüber hinaus weisen die Ergebnisse darauf hin, dass letztlich die Komplexität des gesamten Implementationsgefüges bedeutsam ist: Die Maßnahmen müssen zu den Schüler*innen und zu den individuellen Voraussetzungen der Lehrkräfte sowie den Unterrichtsfächern passen und es bedarf einer Unterstützung durch entsprechende schulische und überschulische Rahmenbedingungen. Konzepte zur Leseförderung sollten beispielsweise als umfassendes Lesecurriculum Teil schulischer Gesamtstrategien und integraler Bestandteil unterrichtlicher Textarbeit in allen Fächern werden. Erforderlich ist daher eine langfristig angelegte Implementation. Dabei benötigen Lehrkräfte zur Umsetzung von Innovationen eine angemessene Entlastung und Unterstützung in Form von Materialien, Fortbildungen und kontinuierlichem Feedback. In einem Projekt wie dem hier vorgestellten Evaluationsvorhaben wird zudem deutlich, wie wichtig eine enge Kooperation zwischen allen Akteur*innen aus Schule, Bildungsadministration und Wissenschaft wäre, damit der Transfer evidenzbasierter Konzepte in die schulische Praxis gelingen kann.

Förderung Das Projekt wird als Teil der gemeinsamen Initiative „Bildung durch Sprache und Schrift (BiSS)“ von BMBF, BMFSFJ, KMK und JFMK gefördert vom Bundesministerium für Bildung und Forschung (BMBF) unter den Förderkennzeichen: 01JI1506A \& 01JI1506B.

Funding Open Access funding enabled and organized by Projekt DEAL.

Open Access Dieser Artikel wird unter der Creative Commons Namensnennung 4.0 International Lizenz veröffentlicht, welche die Nutzung, Vervielfältigung, Bearbeitung, Verbreitung und Wiedergabe in jeglichem Medium und Format erlaubt, sofern Sie den/die ursprünglichen Autor(en) und die Quelle ordnungsgemäß nennen, einen Link zur Creative Commons Lizenz beifügen und angeben, ob Änderungen vorgenommen wurden.

Die in diesem Artikel enthaltenen Bilder und sonstiges Drittmaterial unterliegen ebenfalls der genannten Creative Commons Lizenz, sofern sich aus der Abbildungslegende nichts anderes ergibt. Sofern das betreffende Material nicht unter der genannten Creative Commons Lizenz steht und die betreffende Handlung nicht nach gesetzlichen Vorschriften erlaubt ist, ist für die oben aufgeführten Weiterverwendungen des Materials die Einwilligung des jeweiligen Rechteinhabers einzuholen.

Weitere Details zur Lizenz entnehmen Sie bitte der Lizenzinformation auf http://creativecommons.org/ licenses/by/4.0/deed.de.

\section{Literatur}

Albin, R. W., Lucyshyn, J. M., Horner, R. H., \& Flannery, K. B. (1996). Contextual fit for behavior support plans: a model for 'Goodness of fit'. In L. K. Koegel, R. L. Koegel \& G. Dunlag (Hrsg.), Positive behavioral support: including people with difficult behavior in the community (S. 81-89). Baltimore: Paul H. Brooke.

Altrichter, H., \& Posch, P. (Hrsg.). (1996). Mikropolitik der Schulentwicklung. Förderliche und hemmende Bedingungen für Innovationen in der Schule. Innsbruck: Studien. 
Berkemeyer, N., Bos, W., Manitius, V., \& Müthig, K. (Hrsg.). (2008). Unterrichtsentwicklung in Netzwerken: Konzeptionen, Befunde, Perspektiven. Münster: Waxmann.

Bollen, K. A., \& Curran, P. J. (2006). Latent curve models: a structural equation perspective. New York: Wiley.

Briesch, A. M., Chafouleas, S. M., Neugebauer, S. R., \& Riley-Tillman, T. C. (2013). Assessing influences on intervention implementation: revision of the usage rating profile-intervention. Journal of School Psychology, 51, 81-96.

Bürgermeister, A., Kampa, M., Rakoczy, K., Harks, B., Besser, M., Klieme, E., Blum, W., \& Leiss, D. (2011). Dokumentation der Befragungsinstrumente des Laborexperimentes im Projekt „Conditions and Consequences of Classroom Asessment" ( $\left.\mathrm{Co}^{2} \mathrm{CA}\right)$. Frankfurt a. M.: Deutsches Institut für Internationale Pädagogische Forschung.

Chafouleas, S. M., Briesch, A. M., Riley-Tillman, T. C., \& McCoach, D. B. (2009). Moving beyond assessment of treatment acceptability: An examination of the factor structure of the Usage Rating Profile-Intervention (URP-I). School Psychology Quarterly, 24(1), 36.

Clarke, D., \& Hollingsworth, H. (2002). Elaborating a model of teacher professional growth. Teaching and Teacher Education, 18, 947-967.

Cohen, J., Cohen, P., West, S. G., \& Aiken, L. S. (2003). Applied multiple regression/correlation analysis for the behavioral sciences. Mahwah: Erlbaum.

Dignath, C., \& Büttner, G. (2008). Components of fostering self-regulated learning among students. A meta-analysis on intervention studies at primary and secondary school level. Metacognition and Learning, 3, 231-264.

Elliott, S.N. (1988). Acceptability of behavioral treatments: review of variables that influence treatment selection. Professional Psychology: Research and Practice, 19(1), 66-80.

Firnges, C., \& Steffan, E. (2010). Evaluation von Projekten zur schulischen Qualifikation junger Drogenabhängiger. Berlin: SPI Forschung GmbH.

Goldenbaum, A. (2013). Implementation von Schulinnovationen. In M. Rürup \& I. Bormann (Hrsg.), Innovationen im Bildungswesen. Analytische Zugänge und empirische Befunde (S. 149-172). Wiesbaden: Springer VS.

Graham, J.W. (2009). Missing data analysis: making it work in the real world. Annual review of psychology, 60, 549-576.

Gräsel, C. (2010). Stichwort: Transfer und Transferforschung im Bildungsbereich. Zeitschrift für Erziehungswissenschaft, 13(1), 7-20.

Hasselhorn, M., Köller, O., Maaz, K., \& Zimmer, K. (2014). Implementation wirksamer Handlungskonzepte im Bildungsbereich als Forschungsaufgabe. Psychologische Rundschau, 65, 140-149.

Hattie, J. (2009). Visible learning. A synthesis of over 800 meta-analyses relating to achievement. New York: Routledge.

Hebbecker, K., Meudt, S.-I., Schütze, B., \& Souvignier, E. (2020). Der Transfer von formativem Assessment in den Leseunterricht der Grundschule: Die vermittelnde Rolle von Akzeptanz, Machbarkeit und Kooperation. Zeitschrift für Pädagogische Psychologie. https://doi.org/10.1024/1010-0652/a000279.

IBM Corp (2019). IBM SPSS Statistics for Windows, Version 26.0. Armonk: IBM Corp.

Jerusalem, M., Drössler, S., Kleine, D., Klein-Heßling, J., Mittag, W., \& Röder, B. (2009). Förderung von Selbstwirksamkeit und Selbstbestimmung im Unterricht Skalen zur Erfassung von Lehrer- und Schülermerkmalen. Berlin: Humboldt-Universität zu Berlin.

Jost, J., Karstens, F., Meudt, S.-I., Schmitz, A., Zeuch, N., \& Souvignier, E. (2018). Ein Förderkonzept im Sekundarbereich: Implementation von Lesestrategien zur Förderung der Selbstregulation beim Lesen. In C. Titz, S. Weber, A. Ropeter, S. Geyer \& M. Hasselhorn (Hrsg.), Konzepte zur Sprachund Schriftsprachförderung umsetzen und überprüfen (S. 146-164). Stuttgart: Kohlhammer.

Kamil, M.L., Borman, G. D., Dole, J., Kral, C. C., Salinger, T., \& Torgesen, J. (2008). Improving adolescent literacy: effective classroom and intervention practices: A Practice Guide (NCEE \#2008-4027). Washington, DC: National Center for Education Evaluation and Regional Assistance, Institute of Education Sciences, U.S. Department of Education.

Karstens, F., Schmitz, A., \& Jost, J. (2019). Was kennzeichnet gutes Material zur Förderung von selbstreguliertem Lesen von Sachtexten im Deutschunterricht? - Analyse exemplarischen Fördermaterials für die Sekundarstufe I unter Einbezug pädagogisch-psychologischer und deutschdidaktischer Perspektiven. leseforum.ch, 1/2019, 1-13.

Klieme, E., Artelt, C., Hartig, J., Jude, N., Köller, O., Prenzel, M., Schneider, W., \& Stanat, P. (Hrsg.). (2010). PISA 2009. Bilanz nach einem Jahrzehnt. Münster: Waxmann. 
Klug, J. (2011). Modeling and training a new concept of teacher's diagnostic competence (Dissertation). Darmstadt: Technische Universität Darmstadt. http://tuprints.ulb.tu-darm-stadt.de/2838/1/16.01. 2012_Dissertation_Julia_Klug.pdf. Zugegriffen: 7. Juni 2019.

Körner, S.C. (2002). Das Phänomen Burnout am Arbeitsplatz Schule - Ein empirischer Beitrag zur Beschreibung des Burnout-Syndroms und seiner Verbreitung sowie zur Analyse von Zusammenhängen und potentiellen Einflußfaktoren auf das Ausbrennen von Gymnasiallehrern (Dissertation). Erfurt: Universität Erfurt.

Kullmann, H. (2009). Lehrerkooperation an Gymnasien - Eine explorative Untersuchung zu Ausprägung und Wirkungen am Beispiel des naturwissenschaftlichen Unterrichts (Dissertation). Duisburg-Essen: Universität Duisburg-Essen.

Lindo, E. J., \& Elleman, A. M. (2010). Social validity's presence in field-based reading intervention research. Remedial and Special Education, 31(6), 489-499. https://doi.org/10.1177/0741932510361249.

Magnusson, C.G., Roe, A., \& Blikstad-Balas, M. (2019). To what extent and how are reading comprehension strategies part of language arts instruction? A study of lower secondary classrooms. Reading Research Quarterly, 54(2), 187-212.

Meudt, S.-I. (2019). Transfer innovativer Unterrichtskonzepte: Analysen von Bedingungen für das Gelingen nachhaltiger Veränderungen in der schulischen Praxis (Dissertation). Münster: Universität Münster.

Muthén, L., \& Muthén, B. O. (2010). Mplus user's guide. Los Angeles: Muthén \& Muthén.

National Institute of Child Health and Human Development (NICHD) (2000). Teaching children to read: an evidence-based assessment of the scientific research literature on reading and its implications for reading instruction. Washington, DC: U.S. Government Printing Office.

O'Donnell, C.L. (2008). Defining, conceptualizing, and measuring fidelity of implementation and its relationship to outcomes in K-12 curriculum intervention research. Review of Educational Research, 78, 33-84.

Paulus, C. (2009). Die „Bücheraufgabe“ zur Bestimmung des kulturellen Kapitals bei Grundschülern. http://psydok.psycharchives.de/jspui/bitstream/20.500.11780/3344/1/BA_Artikel.pdf. Zugegriffen: 5. Febr. 2021.

Philipp, M., \& Souvignier, E. (Hrsg.). (2016). Implementation von Lesefördermaßnahmen. Perspektiven auf Gelingensbedingungen und Hindernisse. Münster: Waxmann.

Prenzel, M. (2010). Geheimnisvoller Transfer? Wie Forschung der Bildungspraxis nützen kann. Zeitschrift für Erziehungswissenschaft, 13(1), 21-37.

Proctor, E. K., Landsverk, J., Aarons, G., \& Chambers, D. (2009). Implementation research in mental health services: an emerging science with conceptual, methodological, and training challenges. $A d$ ministration and Policy in Mental Health and Mental Health Services Research, 36, 24-34.

Schellenbach-Zell, J. (2009). Motivation und Volition von Lehrkräften in Schulinnovationsprojekten (Dissertation). Wuppertal: Bergische Universität Wuppertal.

Schermelleh-Engel, K., Moosbrugger, H., \& Müller, H. (2003). Evaluating the fit of structural equation models: tests of significance and descriptive goodness-of-fit measures. Methods of Psychological Research Online, 8(2), 23-74.

Schlagmüller, M., \& Schneider, W. (2007). WLST-7-12. Würzburger Lesestrategie-Wissenstest für die Klassen 7 bis 12. Göttingen: Hogrefe.

Schmellentin, C. (2018). Gedanken zur Implementierung von Sprachbewusstem (Fach-)Unterricht. In B. Hövelbrinks, I. Fuchs, D. Maak, T. Duan \& B. Lütke (Hrsg.), Der-Die-Daz - Forschungsbefunde zu Sprachgebrauch und Spracherwerb von Deutsch als Zweitsprache (S. 121-136). Berlin: De Gruyter.

Schmitz, A., Zeuch, N., Karstens, F., Meudt, S.-I., Jost, J., \& Souvignier, E. (2021). Leseförderung im Schul- und Unterrichtsalltag implementieren - Erste Erkenntnisse des Evaluationsprojekts BiSS-EILe. In S. Gentrup, S. Henschel, K. Schotte, L. Beck \& P. Stanat (Hrsg.), Sprach- und Schriftsprachförderung wirksam gestalten: Evaluation umgesetzter Konzepte (S. 201-220). Stuttgart: Kohlhammer.

Schneider, W. (2019). Programme zur Förderung kognitiver Fähigkeiten in Vorschule und Schule: Wie effektiv sind sie, und wie gut sind die Verfahren praktisch implementiert? Zeitschrift für Pädagogische Psychologie, 33, 5-16.

Slavin, R.E., Cheung, A., Groff, C., \& Lake, C. (2008). Effective reading programs for middle and high schools: a best-evidence synthesis. Reading Research Quarterly, 43, 290-322.

Souvignier, E. (2020). Von der Interventionsforschung zur Implementation. In M. Grosche, J. Decristan, K. Urton, G. Bruns, B. Ehl \& N. C. Jansen (Hrsg.), Sonderpädagogik und Bildungsforschung - Fremde Schwestern? (S. 353-357). Bad Heilbrunn: Klinkhardt. 
Souvignier, E., Jost, J., Schmitz, A., Zeuch, N., Karstens, F., \& Meudt, S.-I. (2020). Bildung durch Sprache und Schrift - Evaluation der Implementation von Konzepten der Leseförderung in der Sekundarstufe I (BiSS-EILe) (Version 1) [Datensatz]. Berlin: IQB - Institut zur Qualitätsentwicklung im Bildungswesen.

Souvignier, E., Trenk-Hinterberger, I., Adam-Schwebe, S., \& Gold, A. (2008). Frankfurter Leseverständnistest für 5. und 6. Klassen. Göttingen: Hogrefe.

Teerling, A., \& Köller, O. (2019). Implementationsprozesse in Schulen: Herausforderungen und Perspektiven. Psychologie in Erziehung und Unterricht, 66, 3-5.

Weiss, R.H. (2006). CFT 20-R: Grundintelligenztest Skala 2 - Revision. Göttingen: Hogrefe.

Wickstrom, K.F., \& Witt, J.C. (1993). Resistance within school-based consultation. In J. E. Zins, T. R. Kratochwill \& S. N. Elliott (Hrsg.), Handbook of consultation services for children: applications in educational and clinical settings (S. 159-177). San Francisco: Jossey-Bass.

Wimmer, H., \& Mayringer, H. (2014). SLS 2-9. Salzburger Lese-Screening für die Schulstufen 2-9. Göttingen: Hogrefe.

Zeuch, N., Schmitz, A., Karstens, F., Meudt, S.-I., Souvignier, E., \& Jost, J. (2018). EILe. Evaluation der Implementation von Konzepten zur Leseförderung in der Sekundarstufe. In S. Henschel, S. Gentrup, L. Beck \& P. Stanat (Hrsg.), Projektatlas Evaluation. Erste Ergebnisse aus den BiSS-Evaluationsprojekten (S. 54-57). Berlin: BiSS-Trägerkonsortium.

Zimmerman, B.J. (2000). Attaining self-regulation: a social cognitive perspective. In M. Boekaerts, P. R. Pintrich \& M. Zeidner (Hrsg.), Handbook of self-regulation (S. 13-39). San Diego: Academic Press. 